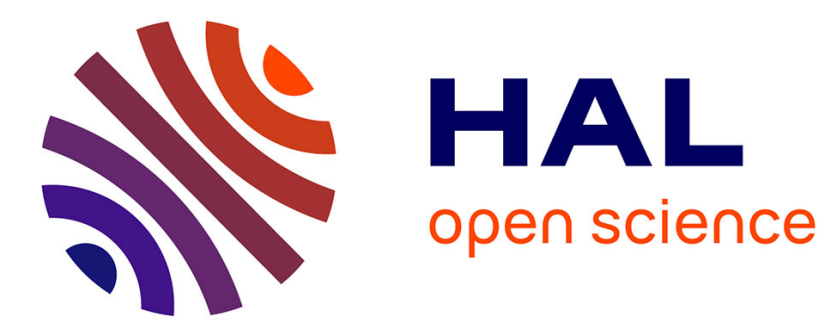

\title{
Vortex kinematics around a submerged plate under water waves. Part II: Numerical computations
} Grégory Pinon, Gaële Perret, Lei Cao, Adrien Poupardin, Jerôme Brossard, Elie Rivoalen

\section{- To cite this version:}

Grégory Pinon, Gaële Perret, Lei Cao, Adrien Poupardin, Jerôme Brossard, et al.. Vortex kinematics around a submerged plate under water waves. Part II: Numerical computations. European Journal of Mechanics - B/Fluids, 2017, 65, pp.368 - 383. 10.1016/j.euromechflu.2016.08.002 . hal-01928816

\section{HAL Id: hal-01928816}

https://hal-normandie-univ.archives-ouvertes.fr/hal-01928816

Submitted on 18 Feb 2021

HAL is a multi-disciplinary open access archive for the deposit and dissemination of scientific research documents, whether they are published or not. The documents may come from teaching and research institutions in France or abroad, or from public or private research centers.
L'archive ouverte pluridisciplinaire HAL, est destinée au dépôt et à la diffusion de documents scientifiques de niveau recherche, publiés ou non, émanant des établissements d'enseignement et de recherche français ou étrangers, des laboratoires publics ou privés. 
European Journal of Mechanics B/Fluids

\title{
Vortex kinematics around a submerged plate under water waves. Part II: Numerical computations
}

\author{
Grégory Pinon ${ }^{\mathrm{a}, *}$, Gaële Perret ${ }^{\mathrm{a}}$, Lei Cao ${ }^{\mathrm{b}}$, Adrien Poupardin ${ }^{\mathrm{c}}$, Jérôme Brossard ${ }^{\mathrm{a}}$, \\ Elie Rivoalen ${ }^{\mathrm{d}, \mathrm{a}}$ \\ a Laboratoire Ondes et Milieux Complexes - UMR 6295, Normandie Univ, UNIHAVRE, CNRS, LOMC, 76600 Le Havre, France \\ ${ }^{\mathrm{b}}$ Université de Technologie de Compiègne (UTC), BP 60319 - rue Roger Couttolenc 60203 Compiègne Cedex, France \\ ${ }^{\text {c } C E A, ~ D A M, ~ D I F, ~ F-91297 ~ A r p a j o n ~ C e d e x, ~ F r a n c e ~}$ \\ d Laboratoire d'Optimisation et Fiabilité en Mécanique des Structures - EA 3828, Normandie Univ, INSAROUEN, LOFIMS, 76800 Saint-Etienne du Rouvray, \\ France
}

\section{A R T I C L E I N F O}

\section{Article history:}

Available online 10 September 2016

\section{Keywords:}

Numerical computations

Submerged plate

Hydrodynamics

Lagrangian vortex method

Water wave

Vortex dynamics

\begin{abstract}
A B S T R A C T
This paper presents numerical computations of the flow generated by a horizontal plate immersed in a regular wave field, and associated loads acting on the plate. This numerical work is the continuation of the Poupardin et al. (2012) experimental study. This numerical study is original in the way that the vortical aspects of the flow are not neglected. Therefore, a 2D Lagrangian Vortex method is used as a numerical scheme. These methods are particularly well suited for the computation of unsteady and highly rotational flows in an open domain. The velocity field is decomposed into rotational and potential components, using the Helmholtz decomposition. The rotational part of the velocity is calculated by the Biot-Savart equation using vortex particles. The plate is modelled by a distribution of normal dipoles and the wave field is taken into account by means of a Stokes formulation, which completes the potential part of the velocity.

Firstly, the numerical code is validated by means of comparison with the experimental results of Poupardin et al. (2012). In particular, the complex vortical activity and the mean flow velocity field are well reproduced and physically analysed. Secondly, forces acting on the plate are analysed on a wide range of parameters by varying the plate immersions and lengths. In the end, a new scaling is found for the lift forces acting on the plate based on the modified Stokes velocity (i.e. the bottom Stokes velocity for a water depth equals to the plate immersion) and the square of the plate length.
\end{abstract}

\section{Introduction}

The interaction of waves with a submerged plate is an important topic widely treated in scientific literature. Such structures were firstly considered for coastal protection properties. Indeed, as they are suspended in the middle of the water column, they have the advantage of preserving the marine environment continuity since they do not directly lie on the seabed. Furthermore, in terms of design, flat plates are more economical and more particularly suited in deep water zones.

Thus, the reflective properties of horizontal submerged plates were firstly studied using the linear potential flow theory [1-3]. Analytical models gave good estimates of the reflection and trans-

\footnotetext{
* Corresponding author.

E-mail address: gregory.pinon@univ-lehavre.fr (G. Pinon).
}

mission coefficients but could not describe the non-linear components of waves and the dynamics of vortices generated at the plate edges. Thus, more sophisticated physical and numerical models were necessary. Patarapanich and Cheong [4] showed experimentally that submerged plates cause the reflection and the dissipation of part of the wave energy. Brossard et al. [5] characterised the free harmonics generation downstream of the plate.

As far as vortex dynamics is concerned, it cannot be neglected when considering the flow around the plate. The importance of vortex generation was first observed by Ting and Kim [6] around a submerged rectangular step using physical modelling. Their measurements, performed with a Laser Doppler Anemometer (LDA) technique, showed the generation of clockwise and counterclockwise vortices periodically shed on both sides of a submerged step. According to their study, these vortices are greatly involved in the dissipation process of the waves. In a similar configuration of submerged rectangular step, Chang et al. also observed vortices for solitary [7] and cnoidal [8] incident waves. In their study, 


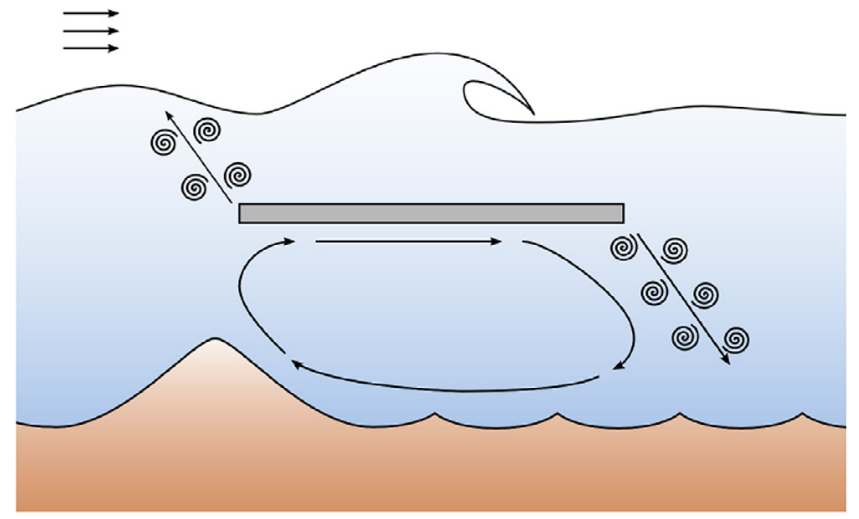

Fig. 1. Schematic representation of the wave flow around a submerged horizontal flat plate in realistic configuration. Reproduction from Cherfils et al. [10] with the kind authorisation of the authors. The wave field propagates from left to right, vortices are generated at the plate's edges and may interact with the free-surface or the seabed. The free surface is modified (wave transmission and wave reflection), recirculation zones may appear as well as scour.

comparisons are made between numerical computations using an unsteady RANS model and experimental measurements with a Particle Image Velocimetry (PIV) technique. They showed that, in the case of a solitary wave propagating over a completely submerged rectangular obstacle, the energy dissipation reaches $15 \%$ of the incident wave energy. In fact, the generated vortices cause an energy cascade of turbulence from large scales towards smaller scales until the viscous dissipation scales. If the vortices strength is still large enough when they reach the seabed, they can generate scour and impact fauna and flora by increasing turbidity. Such vortices may also impact the free surface as shown by Lin et al. [9] who studied the interaction between a submerged vertical plate and a solitary wave by means of PIV measurements. In particular, they observed the appearance of a shear layer between two fluids areas of both sides of the plate which leads to the appearance of a pair of vortices impacting the free surface after a few moments.

More recently, Poupardin et al. [11] experimentally characterised the dynamics of vortices generated at the edges of a submerged plate under a regular wave field. In the studied configuration, vortex shedding is shown to be asymmetric (see the schematic representation of Fig. 1). Upstream of the plate, positive and negative vortices are shed simultaneously and move upstream whereas at the downstream edge, both vortices are advected towards the bottom of the flume. Such vortices clearly indicate the presence of lifting forces, which can affect the design of submerged structures. These loads were quantified experimentally by Rey et al. [12] for a horizontal plate in deep water conditions.

However, few numerical studies were dedicated to vortex dynamics and the associated lifting forces on a horizontal plate submitted to waves. In this configuration, Liu et al. [13] used a numerical method, called Desingularized Boundary Integral Equation Method (DBIEM), able to take into account the non-linear free surface evolution. Their numerical results were compared with Brossard et al. [5] and also with their own experimental results. However, they did not take into account the vortical aspects of the flow. Numerical investigations associated with a similar experiment were also carried out by Cherfils et al. [10] using an inviscid weakly compressible SPH approach. The free-surface modification (wave reflection) was well reproduced but the vortices generation was not possible with this first numerical approach. Then using a viscous weakly compressible SPH formulation, Cherfils [14] modelled the free-surface evolution together with the vortices generation. No-slip boundary conditions were enforced on the plate by an

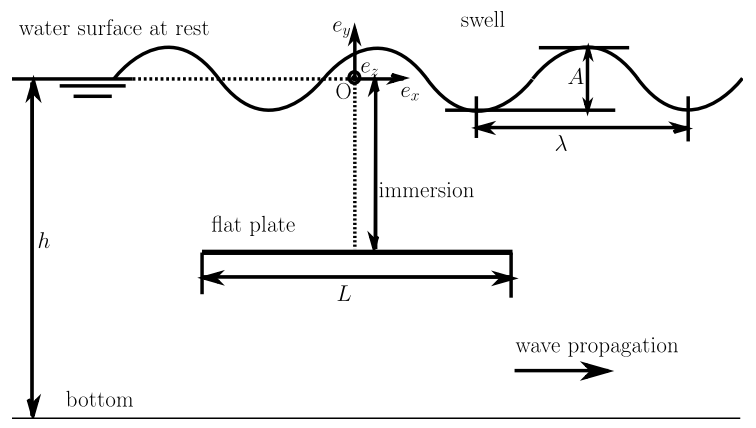

Fig. 2. Schematic representation of the studied geometrical configuration.

innovative Immersed Boundary technique. Unfortunately, the accessible Reynolds numbers were too low to accurately reproduce the vortices dynamics.

The numerical technique presented in this paper is based on Vortex Particles Method performed in a 2D framework. The model is based on the previous work performed by Boulier [15]. The 2D wave field is obtained by a classical Stokes formulation. And the Vortex Method enables to properly model the vortical structures generated at the plate edges. This paper, as the continuation of the work of Poupardin et al. [11], has two main objectives. The first one is to validate the numerical model with their experimental results. The second one is to complete their results by studying the influence of the depth immersion and the plate length on the dynamics and plate loads.

Section 2 presents the numerical scheme. Then, the validation of the model is presented in Section 3 through a comparison of the velocity fields with those obtained experimentally by Poupardin et al. [11]. Forces on the plate are investigated in Section 4 for different depth immersions and plate lengths. Finally conclusions and perspectives are given in Section 5 .

\section{Description of the implemented numerical scheme}

\subsection{From continuous to discretised form of the velocity field}

An horizontal flat plate of length $L$ is immersed in a monochromatic wave train in a 2D configuration, as depicted in Fig. 2. In this paper, bold symbols refer to vectors: for instance, $\mathbf{u}(\mathbf{x}, t)=$ $\left(u_{x}(\mathbf{x}, t), u_{y}(\mathbf{x}, t)\right)$ is the velocity vector for any location in the flow $\mathbf{x}=(x, y)$ at instant $t$. The vorticity field is defined as the curl of the velocity field:

$\omega(\mathbf{x}, t) \mathbf{e}_{z}=\nabla \wedge \mathbf{u}(\mathbf{x}, t)$,

which reduces to a scalar field $\omega(\mathbf{x}, t)$ in 2D. In the following of the paper, space and time dependences are omitted as a matter of writing simplification: for instance, $\mathbf{u}$ will refer to $\mathbf{u}(\mathbf{x}, t)$. The fluid is considered as homogeneous and incompressible, which simplifies the continuity equation (2). Taking the curl of the Navier-Stokes equations, the pressure term vanishes. The viscous part of the Navier-Stokes equations being first omitted (see Appendix B for more details on the diffusion scheme or Refs. [16-18]), the momentum equation basically reduces to Eq. (3) for an incompressible fluid in vorticity-velocity formulation $(\omega, \mathbf{u})$.

$\nabla \cdot \mathbf{u}=0$

$\frac{D \omega}{D t}=0$.

These two equations are the starting point for the 2D Vortex Method. Eq. (3) basically is the transport equation of vorticity $\omega \cdot \frac{D}{D t}$ is the material derivative and $\frac{D \omega}{D t}$ stands for the transport term in a Lagrangian frame. The Helmholtz decomposition of the velocity 
(Eq. (4)) indicates that any vector field can be decomposed as the sum of a rotational component $(\nabla \wedge \psi)$ and a potential one $(\nabla \phi)$, with respectively $\psi$ a vector potential and $\phi$ a scalar potential:

$\mathbf{u}=\nabla \wedge \psi+\nabla \phi$.

The rotational component of the velocity comes from the vorticity field $\omega$, which was defined in Eq. (1), and will be denoted $\mathbf{u}^{\omega}$ such that $\mathbf{u}^{\omega}=(\nabla \wedge \boldsymbol{\psi})$. The potential velocity component represents both the monochromatic wave train owing to the swell $\mathbf{u}^{\mathrm{s}}$ and the influence of the flat plate immersed in the flow $\mathbf{u}^{p}$, such that $\mathbf{u}^{s}+\mathbf{u}^{p}=\nabla \phi$. Owing to these definitions, the total velocity field is the sum of the three following components:

$\mathbf{u}=\mathbf{u}^{s}+\mathbf{u}^{p}+\mathbf{u}^{\omega}$.

In the sequel, a detailed description of each term will be given.

\subsection{Swell formulation}

The swell is considered here as a potential velocity field based on the Stokes formulation in a finite depth [19]. The wave velocity field is then obtained by the following equations:

$$
\left\{\begin{array}{l}
u_{x}^{s}(\mathbf{x}, t)=\frac{\pi A}{T} \frac{\cosh \left(2 \pi\left(\frac{y+h}{\lambda}\right)\right)}{\sinh \left(2 \pi \frac{h}{\lambda}\right)} \cos \left(2 \pi\left(\frac{t}{T}-\frac{x}{\lambda}\right)\right) \\
u_{y}^{s}(\mathbf{x}, t)=-\frac{\pi A}{T} \frac{\sinh \left(2 \pi\left(\frac{y+h}{\lambda}\right)\right)}{\sinh \left(2 \pi \frac{h}{\lambda}\right)} \sin \left(2 \pi\left(\frac{t}{T}-\frac{x}{\lambda}\right)\right),
\end{array}\right.
$$

where $A$ stands for the wave height between peak and trough, $T$ the wave period, $h$ the water depth at rest and $\lambda$ the wavelength, as described on Fig. 2.

\subsection{Velocity field owing to the vortical particles}

Introducing the Helmholtz decomposition (Eq. (4)) into the previous definition of the vorticity field $\omega(\mathbf{x}, t)$ (Eq. (1)) yields:

$-\Delta \psi=\omega$

as $\nabla \wedge \nabla \phi \equiv 0$ by definition.

From the previous Poisson equation (7), the rotational component of the velocity $\left(\mathbf{u}^{\omega}=\nabla \wedge \boldsymbol{\psi}\right.$ ) is obtained by the Biot-Savart relation, which reads in a $2 \mathrm{D}$ space $\&$ by the following integral:

$\mathbf{u}^{\omega}(\mathbf{x}, t)=\int_{\S} \omega\left(\mathbf{x}^{\prime}, t\right) \mathbf{e}_{z} \wedge \mathbf{K}_{0}\left(\mathbf{x}-\mathbf{x}^{\prime}\right) d \&\left(\mathbf{x}^{\prime}\right)$.

$\mathbf{K}_{0}(\mathbf{x})$ is the first derivative of the 2D Green kernel, whose formulation can be simply defined as following:

$\mathbf{K}_{0}(\mathbf{x})=\frac{1}{2 \pi} \frac{\mathbf{x}}{\mathbf{x} \cdot \mathbf{x}}$.

The space integral (Eq. (8)) will be discretised in order to have a discrete approximation of the rotational component of the velocity $\mathbf{U}^{\omega}$ [20-22]. The flow domain is discretised into a sum of $N_{\omega}$ vortex filaments of position $\mathbf{X}_{j}\left(j=1, N_{\omega}\right)$. Each $j$-particle corresponds to a $\mathbf{e}_{z}$-oriented filament, whose circulation is $\Gamma_{j} \approx$ $\Gamma\left(\mathbf{X}_{j}\right) \approx \omega\left(\mathbf{X}_{j}\right) d s\left(\mathbf{X}_{j}\right)$ located at $\mathbf{X}_{j}$. From this point, each vortical filament $\left(\mathbf{X}_{j}, \Gamma_{j}\right)$ is referred as free particles by opposition to the attached particles or bound vortices of the following Section 2.4. However, in order to avoid any collapse of the computation if two particles are too close together during the computation, a classical regularisation of the kernel $\mathbf{K}_{0}$ is performed with a smoothing parameter $\varepsilon$ [20-22]. The smoothed Kernel is obtained by a convolution product with any spherical smoothing function. Among the several smoothing functions existing in literature with different orders of approximation [23], a 4th order polynomial

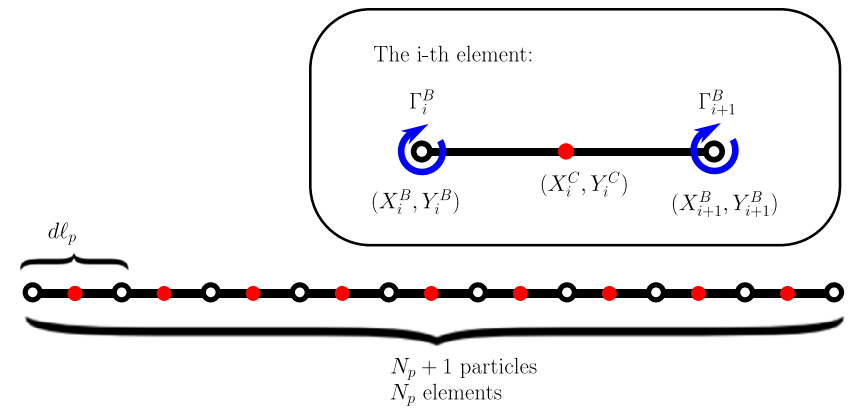

Fig. 3. Schematic representation of the plate discretisation.

function is chosen (see [22, page 27]). This leads to the following regularised kernel $\mathbf{K}_{\varepsilon}$ :

$\mathbf{K}_{\varepsilon}=\frac{\left(r^{4}+3 \varepsilon^{2} r^{2}+4 \varepsilon^{4}\right) \mathbf{x}}{2 \pi\left(r^{2}+\varepsilon^{2}\right)^{3}}$ with $r=|\mathbf{x}|$

as in the previous numerical computations of Boulier et al. [15].

In addition, a global remeshing of the particles is regularly performed [22] in order to maintain an homogeneous distribution of the particles despite their Lagrangian motion. The particles field is remeshed every 7 time steps. The $3^{\text {rd }}$-order $M_{4}^{\prime}$ function (see [22, page 229]) was chosen as a fair compromise between numerical complexity and accuracy. The characteristic length for the global remeshing, which could be named inter-particle's spacing $d r$ is chosen equal to the plate discretisation $d \ell_{p}$ (see following Section 2.4 for details).

Finally, the discrete formulation of $\mathbf{U}^{\omega}$, at a given discrete location in the flow $\mathbf{X}$ and for a given instantaneous time, is:

$\mathbf{U}^{\omega}(\mathbf{X})=\sum_{j=1, N_{\omega}} \Gamma_{j} \mathbf{e}_{z} \wedge \mathbf{K}_{\varepsilon}\left(\mathbf{X}-\mathbf{X}_{j}\right)$

\subsection{Velocity field induced by the flat plate}

The boundary condition on the flat plate is modelled by a distribution of normal dipoles, similarly to the work presented by Nitsche et al. [24,25]. This distribution of normal dipole enforces a free-slip velocity condition onto the plate. Let us consider that the flat plate is divided into $N_{p}$ elements as presented in Fig. 3, the length of each element being constant and equal to $d \ell_{p}$. The free-slip velocity condition is actually enforced at the centre $\left(\mathbf{X}_{i}^{C}\right)_{i=1, N_{p}}$ of each element. As presented in Bousquet [26] for a 2D configuration, a normal dipole on a line element $d \ell_{p}$ is equivalent to two opposite sign vortices positioned at each end of the line element. This finally leads to $N_{p}+1$ bound vortices $\left(\Gamma_{i}^{B}\right)_{i=1, N_{p}+1}$. The discretised velocity $\mathbf{U}^{p}$ induced by these bound vortices is then obtained (Eq. (12)) and is similar to Eq. (11) where the value of $\varepsilon$ is set to zero. This means that the bound vortices induced velocity is not regularised, which is very common using such techniques $[24,25,27]$.

$\mathbf{U}^{p}(\mathbf{X})=\sum_{i=1, N_{p}+1} \Gamma_{i}^{B} \mathbf{e}_{z} \wedge \mathbf{K}_{0}\left(\mathbf{X}-\mathbf{X}_{i}^{B}\right)$

\subsection{Determination of bound vortices distribution}

As mentioned in the previous sub-section, $N_{p}+1$ bound vortices enforces a free-slip velocity condition at the $N_{p}$ element centres 
$\left(\mathbf{X}_{i}^{C}\right)_{i=1, N_{p}}$. In the present configuration, this condition leads to the $N_{p}$ following equations:

$$
\begin{aligned}
& \mathbf{U}^{p}\left(\mathbf{X}_{k}^{C}\right) \cdot \mathbf{n}\left(\mathbf{X}_{k}^{C}\right) \\
& \quad=-\left(\mathbf{U}^{s}\left(\mathbf{X}_{k}^{C}\right)+\mathbf{U}^{\omega}\left(\mathbf{X}_{k}^{C}\right)\right) \cdot \mathbf{n}\left(\mathbf{X}_{k}^{C}\right), \quad k=1, N_{p} .
\end{aligned}
$$

The missing equation is finally obtained by imposing the Kelvin's theorem [28], which can be expressed here as:

$$
\sum_{i=1}^{N_{p}+1} \Gamma_{i}^{B}=-\sum_{j=1}^{N_{\omega}} \Gamma_{j}
$$

where $N_{\omega}$ is the number of free particles in the flow. Finally, a $\left(N_{p}+1\right) \times\left(N_{p}+1\right)$ geometrical matrix system $\overline{\bar{A}}$ is obtained,

$\overline{\bar{A}} \boldsymbol{\Gamma}=\boldsymbol{S M}$

where:

- $\overline{\bar{A}}$ is a geometrical linear matrix,

- $\Gamma$ represents the $\left(N_{p}+1\right)$-vector of unknown circulations of the bound vortices,

- $\boldsymbol{S M}$ is a $\left(N_{p}+1\right)$-vector whose $N_{p}$ first components represent the residual normal velocity component to compensate at $\left(\mathbf{X}_{i}^{C}\right)_{i=1, N_{p}}$ (Eq. (13)), and the last term represents the sum of the $N_{\omega}$ free particles circulation (Eq. (14)).

As the flat plate is fixed during the computation, the matrix $\overline{\bar{A}}$ can be inverted once at the beginning of the computation and its inverse $\overline{\bar{A}}^{-1}$ stored leading to CPU time savings. At each time step, the new distribution of bound vortices is recomputed in order to enforce both free-slip condition (Eq. (13)) and Kelvin's theorem (Eq. (14)). This is obtained by a simple matrix-vector multiplication.

\subsection{Summary of the numerical scheme}

The Lagrangian evolution of the $k$ th-free particles for an incompressible and inviscid fluid is modelled by:

$\begin{aligned} \frac{D \Gamma_{k}}{D t} & =0 \\ \frac{D \mathbf{X}_{k}}{D t} & =\mathbf{U}\left(\mathbf{X}_{k}\right)\end{aligned}$

with

$$
\begin{aligned}
\mathbf{U}\left(\mathbf{X}_{k}\right) & =\mathbf{U}^{s}\left(\mathbf{X}_{k}, t\right)+\mathbf{U}^{p}\left(\mathbf{X}_{k}\right)+\mathbf{U}^{\omega}\left(\mathbf{X}_{k}\right), \\
\mathbf{U}^{p}\left(\mathbf{X}_{k}\right) & =\sum_{i=1, N_{p}+1} \Gamma_{i}^{B} \mathbf{e}_{z} \wedge \mathbf{K}_{0}\left(\mathbf{X}_{k}-\mathbf{X}_{i}\right), \\
\mathbf{U}^{\omega}\left(\mathbf{X}_{k}\right) & =\sum_{j=1, N_{\omega}} \Gamma_{j} \mathbf{e}_{z} \wedge \mathbf{K}_{\varepsilon}\left(\mathbf{X}_{k}-\mathbf{X}_{j}\right)
\end{aligned}
$$

and $\mathbf{U}^{s}\left(\mathbf{X}_{k}, t\right)$ computed with Eq. (6). Eq. (16) interprets the Kelvin's theorem, which represents the conservation of circulation over time for each particle. The particle circulation is not changed as time evolves, only its position is advected due to Eq. (17). A 2ndorder Runge-Kutta is chosen for time stepping scheme. Additionally, in order to model the vorticity shed at the plate edges, particle emission is performed at each time step using the Kutta condition. Some details on the emission procedure are given in Appendix A and the reader can refer to $[29,24,25,30]$ for more details.

The number of involved particles in 2D is not as critical as in 3D computations [31], where acceleration algorithms were implemented: the Treecode algorithm of Lindsay and Krasny [32]. However, parallel computations were performed using the Message Passing Interface (MPI) libraries in order to speed-up the computations.
Table 1

Definition of several numerical parameters including the inter-particle spacing $d r$ the smoothing parameter $\varepsilon$ and the flat plate discretisation $N_{p}$ corresponding to the given element length $d \ell_{p}$.

\begin{tabular}{lllll}
\hline Discretisation & Case 1 & Case 2 & Case 3 & Case 4 \\
\hline$\varepsilon$ & $3.00 \times 10^{-3}$ & $2.50 \times 10^{-3}$ & $2.00 \times 10^{-3}$ & $1.50 \times 10^{-3}$ \\
$d r$ & $2.00 \times 10^{-3}$ & $1.66 \times 10^{-3}$ & $1.33 \times 10^{-3}$ & $1.00 \times 10^{-3}$ \\
$d \ell_{p}$ & $2.00 \times 10^{-3}$ & $1.66 \times 10^{-3}$ & $1.33 \times 10^{-3}$ & $1.00 \times 10^{-3}$ \\
$N_{p}(0.05 \mathrm{~m})$ & 25 & 30 & 38 & 50 \\
$N_{p}(0.10 \mathrm{~m})$ & 50 & 60 & 75 & 100 \\
$N_{p}(0.15 \mathrm{~m})$ & 75 & 90 & 113 & 150 \\
$N_{p}(0.20 \mathrm{~m})$ & 100 & 120 & 150 & 200 \\
$N_{p}(0.25 \mathrm{~m})$ & 125 & 151 & 187 & 250 \\
$d t$ & $5.00 \times 10^{-3}$ & $5.00 \times 10^{-3}$ & $5.00 \times 10^{-3}$ & $5.00 \times 10^{-3}$ \\
\hline
\end{tabular}

Finally, for the configuration described on Fig. 2, in order to impose a free-slip velocity condition at the bottom of the flume, all the particles (either bound or free particles) are symmetrised with respect to the bottom. Fig. 4 depicts the used technique in order to enforce symmetry. The same procedure was also applied for the still water surface (see Fig. 4). In fact, the free surface modifications are not taken into account as previously introduced. In the present study, the ratio $L / \lambda$ was kept to small values $(0.04 \lesssim L / \lambda \lesssim 0.2)$ in order to have from negligible to moderate modifications of the free surface owing to the presence of the plate (cf. Brossard et al. [5]). Thus, the approximation made by neglecting free surface modifications remains valid. Finally, owing to the wish to have a direct ortho-normal vector base (cf. Fig. 2) in the version of the software, the wave propagation is from left to right in the presented computations. This is the opposite sense of propagation with respect to the experimentations described in Poupardin et al. [11]. For that reason, some velocity and vorticity fields presented in Sections 3 and 4 are horizontally mirrored with respect to their experimental counterparts.

\section{Numerical computations and velocity fields}

\subsection{Parameters definition and convergence analysis}

Table 1 presents all the discretisation parameters used in the following computations. The time step $d t$ is set to the same value for all the computation cases. This choice enables us to have the same number of fields for time averaging and to compare unsteady instantaneous field exactly at the same instant. The value $d t=$ $5.00 \times 10^{-3} \mathrm{~s}$ is determined in order to fulfil the CFL like condition for all the tested configurations.

In order to validate the numerical model, geometrical and physical parameters are fixed and identical to those defined in Poupardin et al. [11] study. Therefore, the plate is $L=0.25 \mathrm{~m}$ long, submerged at $i=0.07 \mathrm{~m}$ from the still water level and the water depth is $h=0.2 \mathrm{~m}$. The wave characteristics are the following: wave height $A=0.022 \mathrm{~m}$, period $T=1 \mathrm{~s}$ leading to an incident wavelength $\lambda=1.21 \mathrm{~m}$. In the following of the paper, all the computations were run over 10 periods $(10 T)$. To improve the reliability of the numerical model, numerical computations are also compared to experiments conducted with a smaller plate of length $L=0.05 \mathrm{~m}$ [33], all other parameters being equal. In both experimental cases, a vortical activity was observed around the plate but these vortices were destroyed after two or three wave periods at maximum. However, 2D Vortex Method computations are very conservative. Although, this conservation property is numerically appreciated, it could lead to very different flow patterns from the experimentally observed ones. In fact, although the experiments were performed in a 2D configuration, the shed vortices experienced 3D deformation leading to their explosion (see [33]). These 3D deformations, owing to vortex stretching, could not be taken into account in this 2D approach. Therefore, the numerical 


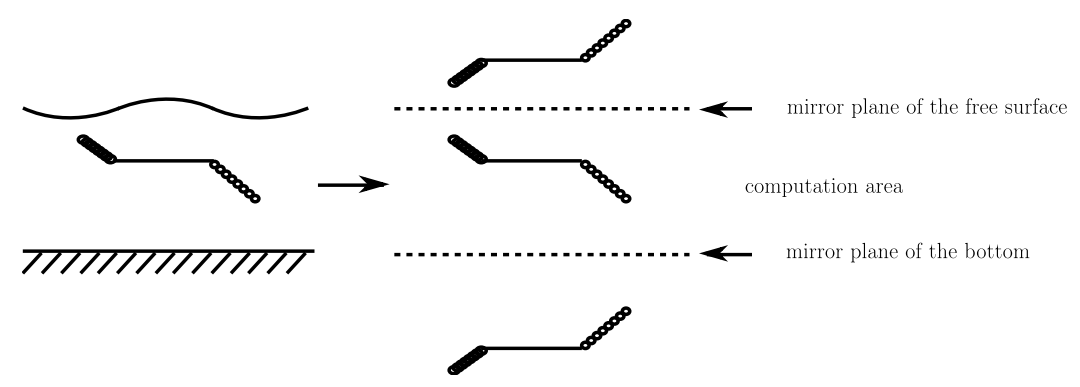

Fig. 4. Schematic representation of the bottom and free surface mirroring process.

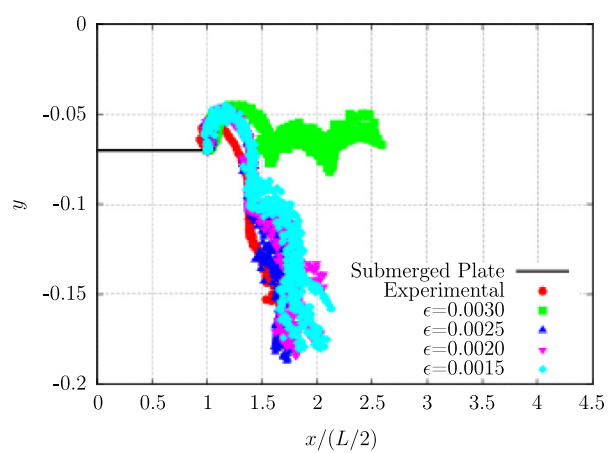

(a) $L=0.25 \mathrm{~m}$.

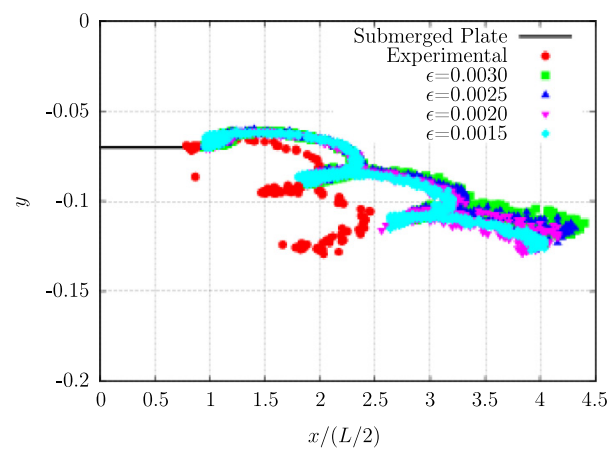

(b) $L=0.05 \mathrm{~m}$.

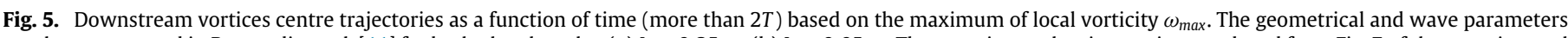

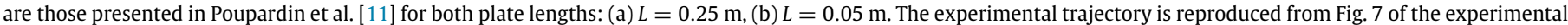
study [11] and from Ref. [33] for the smaller plate. The four discretisations are presented in Table 1.

model described in Section 2 needed to be improved to first, account for diffusion and second, attempt to model dissipation. Appendix $B$ presents the chosen numerical diffusion procedure and how this model was modified to account for dissipation.

Fig. 5 depicts the downstream vortices trajectories as a function of time for both plate length configurations. The trajectories are compared with the experimental ones for different values of the smoothing parameter $\varepsilon$. It can easily be observed that the coarsest discretisation $(\varepsilon=0.0030)$ poorly models the downstream vortex trajectory for the 0.25 m-length plate configuration. Refining the resolution increases the quality of the numerically computed trajectories. Appendix B presents additional results comparing the numerical and experimental results. Based on trajectories comparison (see Fig. 5 and Appendix B), the numerical tool can be considered as validated. The smallest discretisation of Table 1 is chosen for all the following computations together with the use of a diffusion and dissipation models as described in Appendix B. Physical analysis of the velocity and vorticity fields will now be presented in the following section.

\subsection{Vortical and velocity fields}

Fig. 6 shows the vorticity field evolution as a function of time for 8 different phases within a wave period $T$. The vortical activity experimentally observed in the previous work of Poupardin et al. [11] is well reproduced with this numerical model. In the lee-side, at each wave period $T$, an opposite sign pair of vortices is created and advected downward owing to mutual induction. Two to three pairs of vortices can be identified, depending on the given phase inside the wave period. The vortex generation is very similar to the one observed on figures Fig. 6(a), (b) and (c) of Ref. [11]. As discussed earlier, the vortices trajectories are rather well reproduced (see Figs. 5 and B.16 of Appendix B) with these parameters. However, in the numerical results, the vortices persist a little longer than experimentally. An additional possible explanation is that the vortices are destroyed through 3D instabilities in the experiments, which cannot be reproduced in the present 2D numerical configuration.

An interesting feature is that these vortex pairs regularly hit the seabed producing, in real configuration, a zone of possible scour. Indeed, while impacting the seabed, the vortices separate each other, one eventually going downstream and the other upstream. This creates a shear flow at this bottom location of the wave flume. In real-life configuration, either at the lab scale or in situ, this shear flow leads to the generation of vorticity. The generated vorticity undoubtedly feeds in the steady recirculation zone, already identified in Ref. [11] on the mean velocity field. Therefore, the numerically computed average velocity field will now be studied.

Fig. 7 depicts the numerically computed steady flow as an average of 200 instantaneous fields representing 5 wave periods $T$. The non-dimensional coordinates were chosen so as to get a similar representation as Fig. 3 of Ref. [11]. The jet-like flow (also referenced as "C" on Fig. 3 of Ref. [11]) is easily observable on the numerically computed flow of Fig. 7 . The stagnation point identified as " $D$ " in the previous reference is also identifiable, even though slightly shifted downward at $x /(L / 2)=1.84$ in comparison to $x /(L / 2)=1.5$ experimentally. To some extent, the differences can clearly be attributed to the weakness of the numerical steady recirculation cell " $A$ ", which is not easily observable on Fig. 7. However, it is striking to see that the other recirculation cell identified as "B" on both graphs of Fig. 3 (Ref. [11]) and Fig. 7 are very similar. The centre of this recirculation cell is approximately located at $(-1.44,0.69)$ numerically which is very comparable to the $(-1.38,0.64)$ values observed experimentally. Additionally, another smaller recirculation cell is numerically observed right above the upstream end of the plate as in the experiments. Moreover, the stagnation point (zone "E" of Fig. 3, Ref. [11]) experimentally observed under the plate is also numerically reproduced although its location is not exactly the same. This discrepancy is possibly due to the shift and/or weakness of the recirculation cell " $A$ ". However, generally speaking, this 

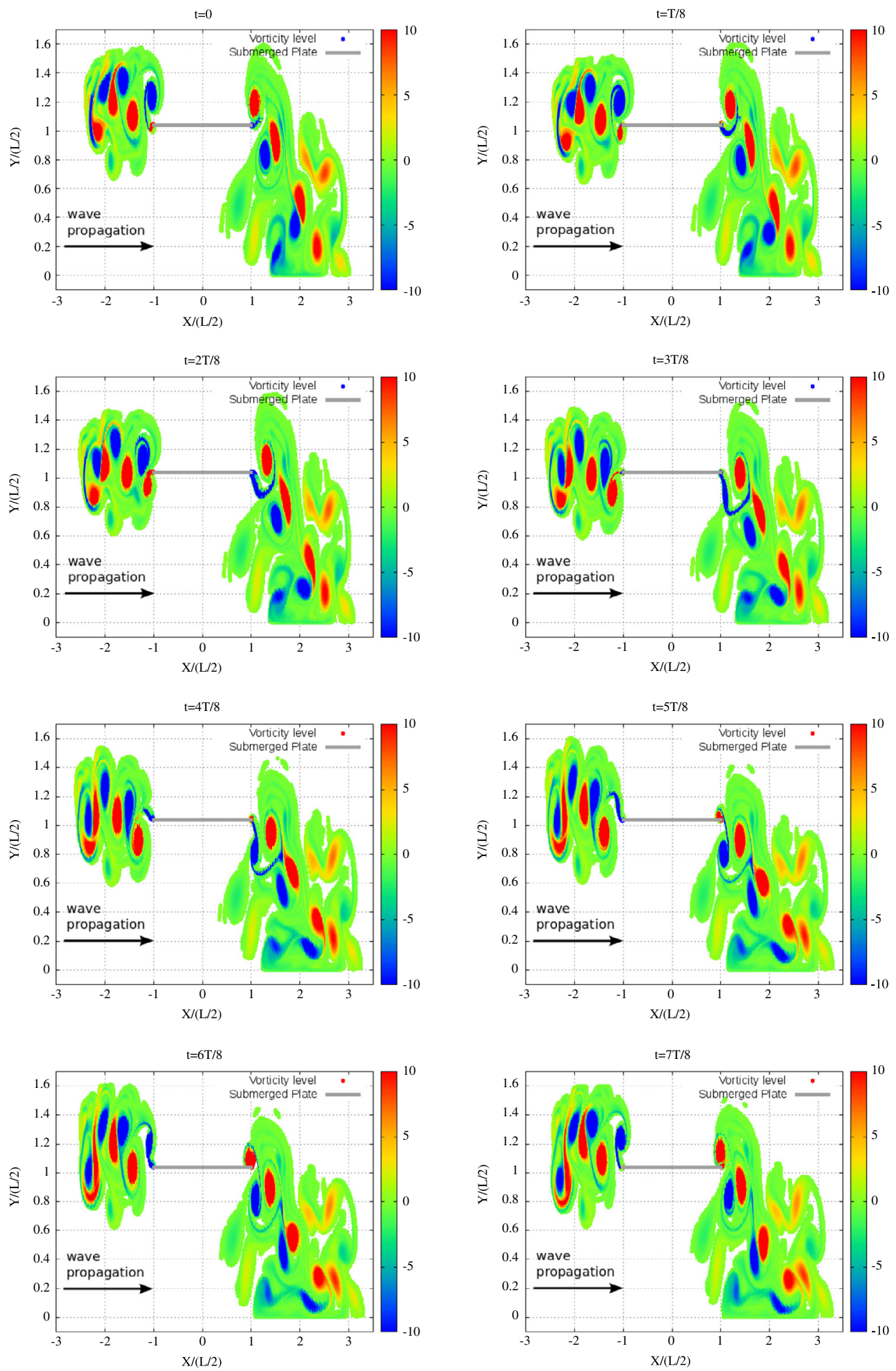

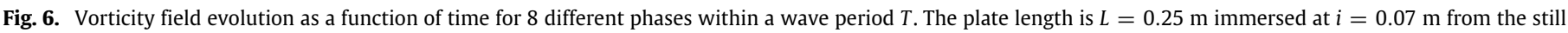
water level. The discretisation is $\varepsilon=0.0015$ as presented in Table 1 .

confirms that placing a rotor at this precise location will probably not produce energy, at least not as much as the potential flow theory would suggest [34-37].

\subsection{The influence of the large lee-side recirculation cell}

The influence of the large lee-side recirculation cell identified as "A" on Figure 3 of Poupardin et al. [11] is important in order to interpret the whole flow pattern. While the jet-like flow is impacting the seabed experimentally, vorticity is created. The bottom upward flow observed on Fig. 3 of Ref. [11] permanently feeds in this recirculation cell "A". In the present numerical implementation, as a free-slip boundary condition was chosen, no vorticity generation is observed at this location. The bottom upward flow can be numerically observed on Fig. 7 but without vorticity generation. 


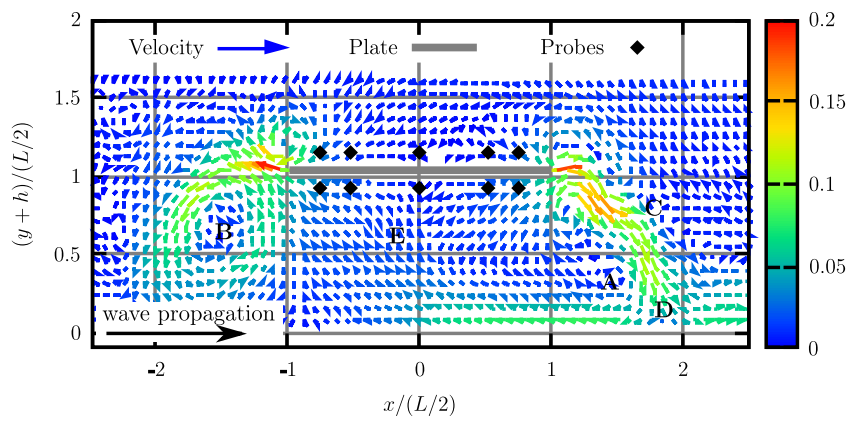

Fig. 7. Averaged velocity field over 200 instantaneous velocity fields representing 5 wave periods $T$. The plate length is $L=0.25 \mathrm{~m}$ immersed at $i=0.07 \mathrm{~m}$ from the still water level. The discretisation is $\varepsilon=0.0015$ as presented in Table 1 .

In order to assess the influence of this large lee-side recirculation cell, an additional fixed particle $\left(\mathbf{X}_{f p}, \Gamma_{f p}\right)$ was added precisely at the location of the experimentally observed recirculation cell " $A$ ". The velocity of this fixed particle is basically computed with Eq. (11) of Section 2.3. This computed velocity was added to the other velocity components of the Helmholtz decomposition (Eq. (18)) but the numerical implementation is not further modified. The advantage of such a simple implementation is that it steadily mimics the influence of this experimentally observed recirculation cell " $A$ " without the need of a fully resolved boundary layer, which would be computationally expensive. The drawbacks are that this fixed particle circulation is user defined and needs to be known a priori. Additionally, the fixed particle influence is constant over a wave period unless its position and circulation could be adjusted over time. But this would need much more experimental information. Finally, this simple procedure cannot be applied for other geometrical configurations than the present experimentally observed one as experimental values are required. However, it is interesting to observe that this simple procedure greatly enhances the lee-side flow pattern with a very low algorithmic and computational cost. In the future, the account of a no-slip boundary condition on the seabed is however worth considering, such as the technique implemented by Ploumhans et al. [38] for instance.

Fig. 8 depicts the $x$-velocity component for the 10 locations presented on Fig. 7. Such comparisons are very instructive and enable to distinguish the physical processes that are correctly reproduced from those that are not. Probes 1 to 5 are located on the upper side of the plate from upstream to downstream respectively. Probes 6 to 10 are located at the lower part from upstream to downstream too. The experimental velocities actually are phaseaveraged velocities from the PIV measurements performed in Poupardin et al. [11] study. The numerical velocities are not phaseaveraged showing a variation depending on the period, more especially for the case with an additional fixed particle. For the case without additional fixed particle, the different numerical velocity profiles quasi super-impose.

The left hand side of Fig. 8 depicts the horizontal velocity evolution for the 5 upper probes. A first observation is that both experimental and numerical velocity evolutions at the plate centre (probe 3) present a quasi symmetric sinusoidal shape. The amplitude of this signal is close to $U=0.069 \mathrm{~ms}^{-1}$, the Stokes velocity at this immersion. As the probe location gets closer to the plate edges, the experimental velocity amplitude increases due to the presence of vortices. Numerically, this velocity amplitude increase is well reproduced at the downstream edge of the plate (probes 4 and 5) meaning that the vortex dynamics seems to be correctly reproduced. At the upstream edge (probes 1 and 2), the numerical velocities qualitatively follows the experimental ones although the amplitude increase is not reproduced at all. A second observation is that the experimental velocities is no more symmetric at the downstream edge of the plate, probably due to the non-linear deformation of the free surface above the plate. This explains the discrepancies observed between the experimental and numerical velocity evolutions for probes 4 an 5 since this non-linear evolution of the free surface cannot be modelled numerically.

The right hand side of Fig. 8 presents the time evolution of velocity for the five lower probes represented on Fig. 7. The five experimental velocity records are all very close to a sinusoidal shape. This sinusoidal shape is centred on probe 8 , located at the centre of the plate, with a velocity amplitude close to characteristic velocity $U$. However, owing to the presence of the steady recirculation cells denoted "A" and " $B$ " on Fig. 3 of the experimental part [11], time evolution of the velocity are either negatively or positively off-set from zero experimentally. For instance upstream, probe 6 and - to a smaller extent - probe 7 indicate a negative off-set which can be attributed to the steady recirculation cell "B". This feature is correctly reproduced numerically, even without additional fixed particle, as the numerical model directly brings out this steady recirculation cell denoted "B" on Fig. 7. For the downstream side, a large positive off-set in the velocity amplitude of nearly $2 U$ is experimentally observed (probes 9 and 10). Numerically, a positive off-set is also observed but with a smaller amplitude than in the experiments. However, velocity evolution fits much more the experimental profile by taking into account the fixed particle $\left(\mathbf{x}_{f p}, \Gamma_{f p}\right)$ placed under the plate. The position of this fixed particle is based on the mean position over one period obtained experimentally, i.e. $x_{f p}=0.108 \mathrm{~m}$ and $y_{f p}=-0.145 \mathrm{~m}$ with an imposed circulation of $\Gamma_{f p}=-0.0015 \mathrm{~m}^{2} \mathrm{~s}^{-1}$.

In this given configuration (water depth, plate length, wavelength and amplitude), these global flow patterns clearly play an important role on the flow modification close to the plate. In fact, the presence of the plate in the wave field first creates an opposite sign pair of vortices which secondly generates a downward jet-like flow. Third, this jet impacts the sea-bottom (cf. Fig. 7) leading to vorticity generation at the flume bottom in real configuration. Fourth, this vorticity finally creates and feeds in a downstream steady recirculation cell (cf. "A" on Fig. 7), which eventually interacts with the local flow around the plate and eventually intensifies the vortices generation at the plate level. This wave-plate interaction finally leads to a complex global flow pattern. The influence of each physical process could be more easily explained with this numerical approach, as it enables to separate them, than through the experiment.

In order to further emphasise the role of the recirculation cell on the flow, the averaged velocity field with the additional fixed particle $\left(\mathbf{X}_{f p}, \Gamma_{f p}\right)$ is plotted on Fig. 9. The reproduced flow features are very similar to those observed Fig. 7 . All the upstream flow patterns remain unmodified: the jet like-flow persists similarly and the recirculation cell " $\mathrm{B}$ " is not significantly modified. The main difference lies in the presence of a clear recirculation cell denoted " $A$ " downstream. This recirculation cell " $A$ " is more accurately reproduced and the impact location " $D$ " is also more accurately predicted with a value of $x /(L / 2)=1.33$ in comparison with $x /(L / 2)=1.5$ experimentally. The averaged flow of Fig. 9 more resembles the experimental one (Fig. 3 of Ref. [11]) than the previous Fig. 7. To conclude, the numerical software is able to reproduce the experimental flow with a good accuracy. This accuracy is even improved with this account of an ad hoc fixed particle to mimic the large lee-side recirculation cell created by vorticity generation at the bottom of the wave flume.

Although the 3D instabilities of these vortices and the generation of vorticity at the bottom cannot be taken into account with the present numerical implementation, the averaged velocity field is well reproduced. 

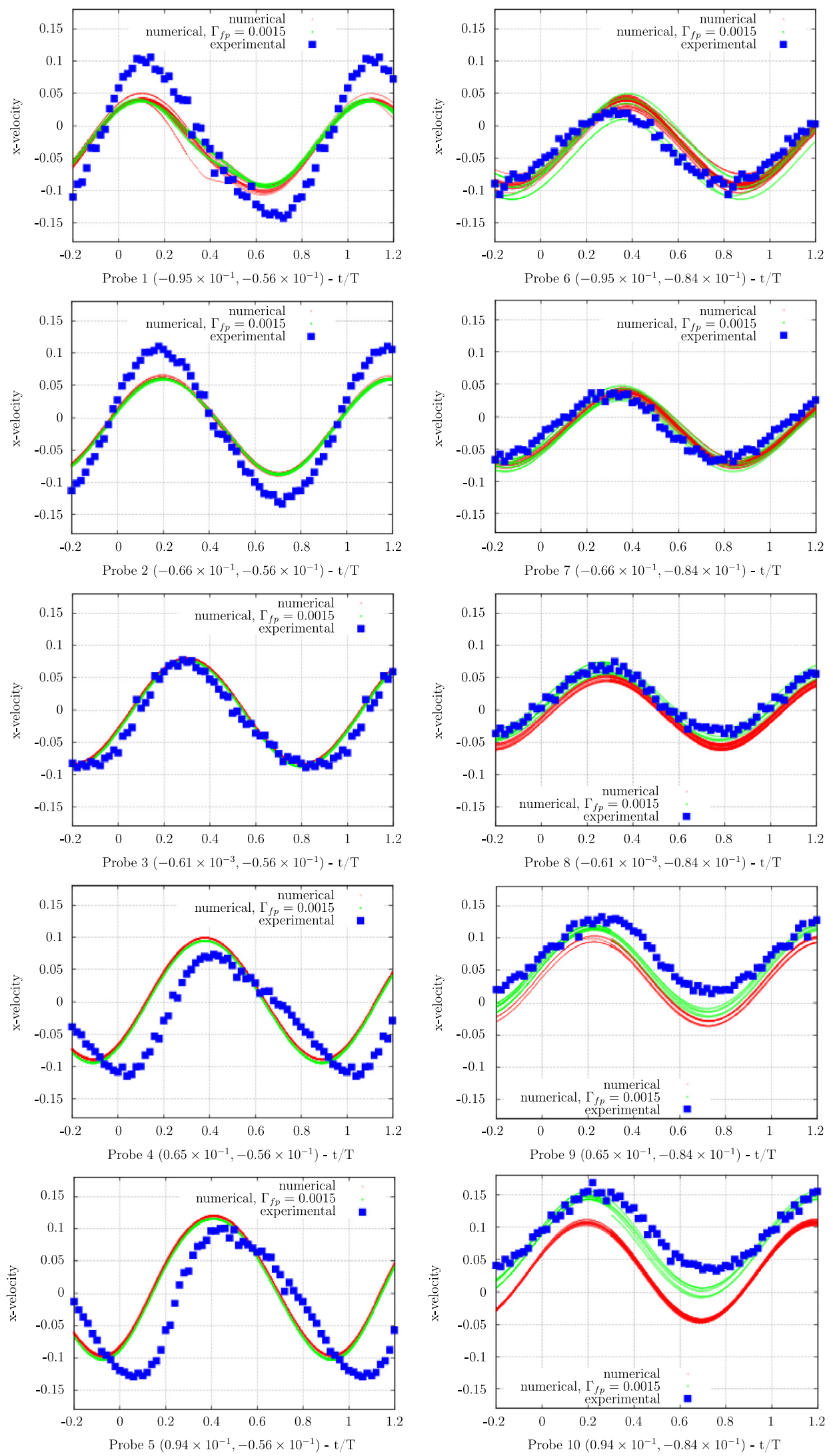

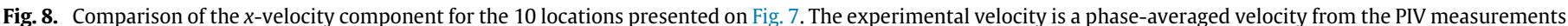

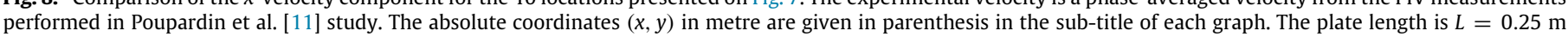
immersed at $i=0.07 \mathrm{~m}$ from the still water level. The discretisation is $\varepsilon=0.0015$ as presented in Table 1 . 


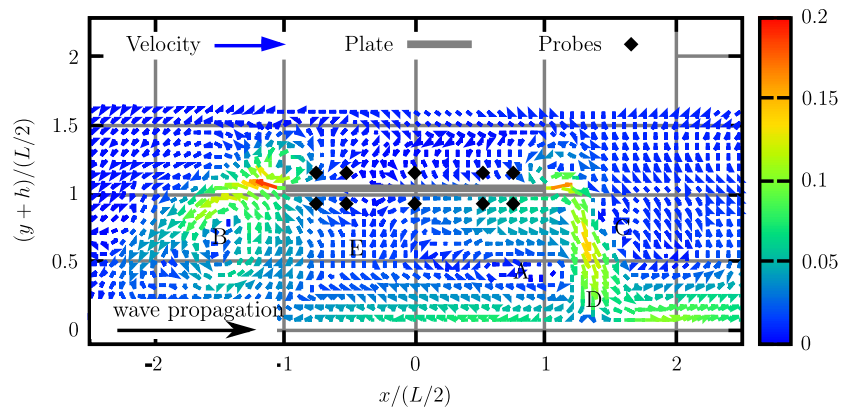

Fig. 9. Averaged velocity field over 200 instantaneous velocity fields representing 5 wave periods $T$ with the account of fixed particle representing the large lee-side recirculation cell. The plate length is $L=0.25 \mathrm{~m}$ immersed at $i=0.07 \mathrm{~m}$ from the still water level. The discretisation is $\varepsilon=0.0015$ as presented in Table 1 .

\section{Force evaluation on the plate}

Following the work of Le Maître et al. [27] dealing with a numerical study of two-dimensional sail in unsteady flow, their expression for the determination of the force acting on the plate will be considered. In their study, the fluid is considered inviscid, incompressible and the sail is supposed to have a very small thickness. Their configuration is very similar to the present one, therefore their study and results will be taken as a reference. According to equation (11) of the above-cited paper [27], the pressure jump $\Delta P$ acting on the thin structure (either a sail or a plate) can be calculated using the following equation:

$\Delta P=\rho\left[\gamma^{B} \mathbf{e}_{z} \wedge \mathbf{U}(\mathbf{x})\right] \cdot \mathbf{n}+\rho \int_{0}^{l} \frac{d \gamma^{B}\left(l^{\prime}\right)}{d t} d l^{\prime}$

where $\gamma^{B}$ represents the vortex sheet strength distribution along the plate. It physically represents the tangential velocity jump across the plate and is therefore homogeneous to $\left[\mathrm{m} . \mathrm{s}^{-1}\right]$. The density $\rho$ is going to be set to unity without any loss of generality and n stands for the plate normal vector. In this Eq. (21), the first term stands for the steady component and the second for the unsteady one. Appendix C presents some details on the discretised form of Eq. (21) and test cases and results used for the validation of the present numerical approach. Finally, the discretised form of the force $\mathbf{F}$ acting on the plate is:

$\mathbf{F}=\sum_{i=1, N_{p}} \Gamma_{i}^{B} \mathbf{e}_{z} \wedge \mathbf{U}\left(\mathbf{X}_{i}^{C}\right)+\sum_{i=1, N_{p}} \sum_{j=1, i} \frac{d \Gamma_{j}^{B}}{d t} d \ell_{p}$,

where the $\Gamma_{i}^{B}$ and $\frac{d \Gamma_{j}^{B}}{d t}$ values are evaluated on $\mathbf{X}_{i}^{C}$.

\subsection{Spatial and temporal evolution of forces acting on the plate}

Fig. 10(a) depicts the pressure distribution over the $L=$ $0.25 \mathrm{~m}$ long flat plate for the eight different phases in the wave period represented on Fig. 6. The considered computation is performed with the finest discretisation $\varepsilon=0.0015$. For this given configuration, the pressure imposed by the vertical Stokes wave velocity component $u_{y}^{s}(t)$ of Eq. (6) is not sufficient to explain the pressure amplitude (and hence, the force) acting on the plate. Indeed, the maximum value $\rho\left(u_{y}^{s}\right)^{2} \approx 1.710^{-3} \mathrm{~Pa}$ is much smaller than the $p \approx 6.010^{-2}$ Pa pressure numerically recorded on the plate (see Fig. 10(a)).

However, the temporal variation of pressure can be explained by the vortical activity surrounding the horizontal plate. For instance, the negative pressure distribution recorded at $t / T=$ 0.875 or $t / T=0$ can be explained by the vorticity fields presented on Fig. 6. In fact, for these two phases a negative vortex is well formed at the upper upstream side of the plate and a positive vortex is also well formed at the upper downstream side. These two vortices indubitably induce the negative bell shape pressure distribution (see Fig. 10 (a) for $t / T=0.875$ and $t / T=0$ ). On the contrary, for $t / T=0.5$ or $t / T=0.625$, the opposite situation can be observed. In fact, a negative vortex is formed at the lower downstream side of the plate whereas a positive one can be found at the lower upstream side (cf. Fig. 6). These two vortices involve a positive pressure distribution on the plate, which is observed on Fig. 10(a). For $t / T=0.25$ or $t / T=0.75$, intermediate situations are also observable: a vortex is generated earlier at one given side of the plate and the pressure distribution starts to reverse. To conclude, the pressure distributions presented on Fig. 10(a) can be explained by the corresponding vortical activity depicted on Fig. 6 .

These instantaneous pressure distributions can be integrated over the plate and the resulting force $F_{y}(t)$ is recorded at each time step. Fig. 10(b) depicts the time evolution of the vertical force component computed from Eq. (21) for the 7 last periods of time over the overall 10 computed periods. As the wave flow is periodic, the recorded force history is also well periodic as observed on Fig. 10(b).

For the $L=0.25 \mathrm{~m}$ long plate, the periodic situation really repeats at each period. The maximum negative force occurs at approximately $t / T=0.9$ and the maximum positive one approximately at $t / T=0.5$, corresponding to the pressure distributions described here-above (Fig. 10(a)). For the $L=0.05 \mathrm{~m}$ long plate, the force history is somehow similar except that the periodic repetition is less clear: more noise is perceivable especially between $t / T=0$ to $t / T=0.4$. Additionally, the maximum normal force recorded is much less than the plate length difference; the force ratio is approximately 25 whereas the plate length ratio is basically 5 . The physical analysis of this vertical force component presented in the previous paragraph needs to be validated against some parameters' variation. This parameters' variation analysis is performed in the following sub-section.

\subsection{Parametric study}

In this parametric study, the five plate lengths presented in Table 1 were tested. All the computations were run with the finest discretisation $\varepsilon=0.0015$ and the corresponding parameters described in Table 1 . The immersion depths are ranging from $i=$ $0.06 \mathrm{~m}$ to $0.15 \mathrm{~m}$ for a total water depth of $h=0.2 \mathrm{~m}$. These limits of $i / h$ were determined by the presence of the upper and lower boundaries. Indeed, out of this range of plate immersion, the vortical activity would not have been accurately computed owing to the presence of the free surface or seabed. The water depth $h$ and incident wavelength $\lambda=1.21 \mathrm{~m}$ were kept constant for all computations.

Fig. 11 depicts several instantaneous vorticity fields recorded at $t / T=0$, seven wave periods after the initialisation of the computation. For readability reasons, only three immersions $i=$ $0.07 \mathrm{~m}, 0.11 \mathrm{~m}$ and $0.15 \mathrm{~m}$ are presented for the five plate lengths. The different graphs depicted on Fig. 11 show that vortex shedding is present for all plate lengths and immersions, although the vortical levels are different. In fact, vortices are weaker for the shorter plates and this tendency is increased for deeper immersions. Apart from these cases, at least two pairs of opposite sign vortices can be identified in the flow for nearly all graphs. The vortex generation presented in Section 3.2 seems to apply for quasi all these cases. Hence, the mechanism responsible for the pressure onto the plate, described in Section 4.1 should apply. A dimensional analysis is performed in the following paragraph in order to explain the pressure level (or force) acting on the plate in a wave field.

To study the parametric evolution of the vertical forces acting on the plate, a time averaging of the force is performed. However, 


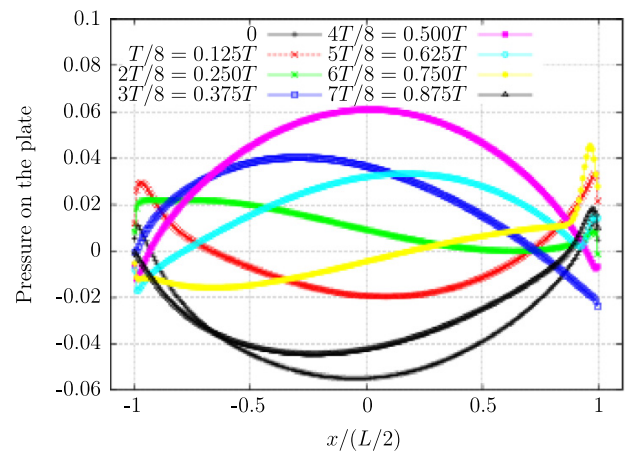

(a) Pressure distribution on the plate.

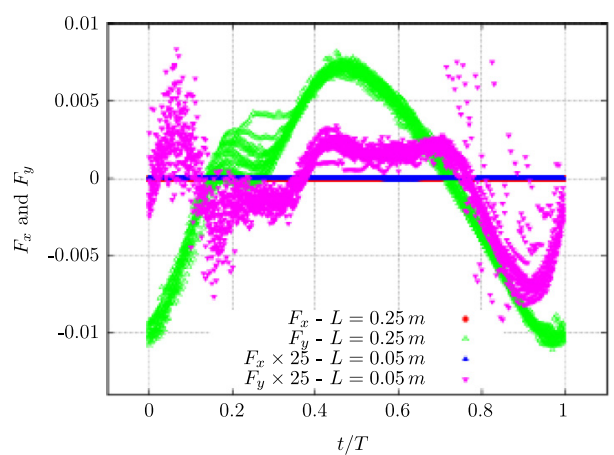

(b) Temporal variation of the force.

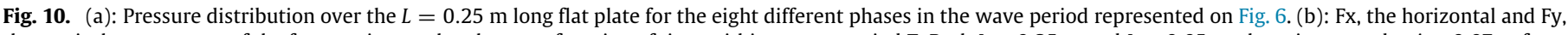

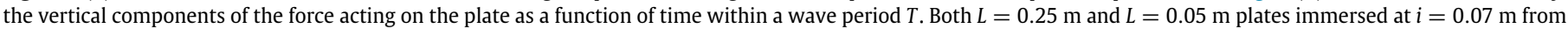
the still water level are considered. The discretisation is $\varepsilon=0.0015$ as presented in Table 1 .

the classical time average value $\overline{F_{y}}$ of $F_{y}(t)$ is close to zero. Although not exactly null, no characteristic force was accessible with this way of averaging. For that reason, an average of the absolute value of $\left|F_{y}(t)\right|$ over the last seven periods of time is performed using the following Eq. (23):

$\overline{\left|F_{y}\right|}=\frac{1}{7 T} \int_{t=3 T}^{t=10 T}\left|F_{y}(t)\right| d t$.

The averaging is performed over the seven last wave periods in order to remove the transient values of the force generated at the beginning of the computations.

Fig. 12 depicts the numerically computed values of $\overline{\left|F_{y}\right|}$ for the different plate lengths and immersions. Two simplistic remarks can be argued from this graph: the longer the plate is, the higher the averaged force $\overline{\left|F_{y}\right|}$; and the deeper the plate's immersion is, the lower the force for a given plate length.

Actually, lifting forces acting on an object can be scaled with a characteristic circulation $\Gamma^{*}$ around this object times a characteristic length scale $L^{*}$ divided by a time scale $T^{*}$. For instance, both terms of Eq. (22) representing the force acting on the plate dimensionally correspond to $\rho \Gamma^{*} L^{*} / T^{*}$. However, as the flow is highly unsteady, one would expect that the steady component (first term of Eq. (22)) would be negligible and in fact the mean force $\overline{F_{y}(t)}$ is null. As a matter of consequence, the main component of the force should be the unsteady part (second term of Eq. (22)) which clearly corresponds to $\rho \Gamma^{*} L^{*} / T^{*}$ dimensionally speaking. And a circulation $\Gamma^{*}$ is basically the product of a characteristic velocity $U^{*}$ times a characteristic length scale $L^{*}$. Using these two scaling procedures, the vertical load acting on the plate should be some function of $\rho U^{*}\left(L^{*}\right)^{2} / T^{*}$. The characteristic length and time scales are undoubtedly the plate length $L$ and the wave period $T$ respectively. An intuitive characteristic velocity would be $u_{x}^{s}$ (Eq. (6)), the horizontal component of the first order Stokes velocity evaluated at immersion depth $y=i$. However, using this characteristic velocity $u_{x}^{s}(x, y=i)$ presented in Table 2 , no clear scaling of the force could be found.

Following the work of Brossard et al. [5], an improved estimate of the characteristic velocity could be made. When propagating over the submerged plate, the incident waves are modified by the presence of the plate. If the plate is long enough, it is as if the flume bottom was actually the upper surface of the plate. The characteristic wavelength of the flow over the plate is then modified. And a modified wavelength $\lambda_{i}$ can be obtained with the Stokes dispersion relation calculated for a water depth equal to the plate immersion $i$ (see Table 2). The characteristic velocity is then chosen as the horizontal Stokes velocity $\left(u_{x}^{s, \lambda_{i}}\right)$ evaluated at the plate immersion (i.e. at the bottom for a water depth equal to the plate immersion $i$ and the corresponding wavelength $\lambda_{i}$ ). The values of $u_{x}^{s, \lambda_{i}}$ are also given in Table 2 .

Fig. 13 depicts the averaged vertical force $\overline{\left|F_{y}\right|}$ as a function of $\rho u_{x}^{s, \lambda_{i}} L^{2} / T$ for all the plate immersions and lengths, in a log-log scale. Except for the smallest plate length $(L=0.05 \mathrm{~m})$, the forces scale with a very good accuracy to the characteristic variables $\rho u_{x}^{s, \lambda_{i}} L^{2} / T$. The obtained residual from the linear regression is 0.9979 which tends to prove that the linear fit is very accurate. This further confirms that $u_{x}^{s, \lambda_{i}}$ seems to be the relevant parameter for dimensioning such immersed structures, leading to:

$\overline{\left|F_{y}\right|} \propto \rho u_{x}^{s, \lambda_{i}} L^{2} / T$.

For the smallest plate length $(L=0.05 \mathrm{~m})$, the wave flow around the plate is much less modified than for the other configurations. It is as if the plate was too small to modify the incident wave field. This observation was already mentioned in the previous work of Brossard et al. [5]. Additionally, the vortical activity is also weaker if compared to the other cases (see the first line of graphs in Fig. 11). For the computations with $L=0.05 \mathrm{~m}$, the present scaling does not fit any more as depicted on Fig. 13. For the two or three smaller immersions, where the vortical activity is still a little present (see top left of Fig. 11), there is a tendency to follow the scaling although there is an offset. As the immersion increases, the concerned points of Fig. 13 completely diverge from the linear fit and no clear scaling could be found using any of the characteristic velocities.

This tends to suggest the existence of threshold for the ratio $L / \lambda$, where $\lambda$ is the incident wavelength, below which the wave characteristics remain unperturbed. For the smaller plate $(L=$ $0.05 \mathrm{~m}), L / \lambda=0.041$ is obtained whereas for $L=0.10 \mathrm{~m}$ this ratio increases to $L / \lambda=0.08$. Thus, the threshold should be situated between those two values. However, a future investigation comprising experimental and numerical tests is required in order to fully validate the obtained results. In fact, these forces were determined without the account of the large lee-side recirculation cell as presented in Section 3.3. It is anticipated that the increased velocity around the plate, as depicted in Fig. 8, will further increase the vortical activity and hence the force undergone by the plate.

\section{Conclusion}

This study presents the numerically computed flows generated by a monochromatic incident wave interacting with an immersed horizontal plate. This paper follows and completes the experimental study of Poupardin et al. [11]. On the one hand, the experimental results are used to validate the numerical model in the same test conditions. On the other hand, the control parameters are varied to study the evolution of the flow and the forces on the plate. The 

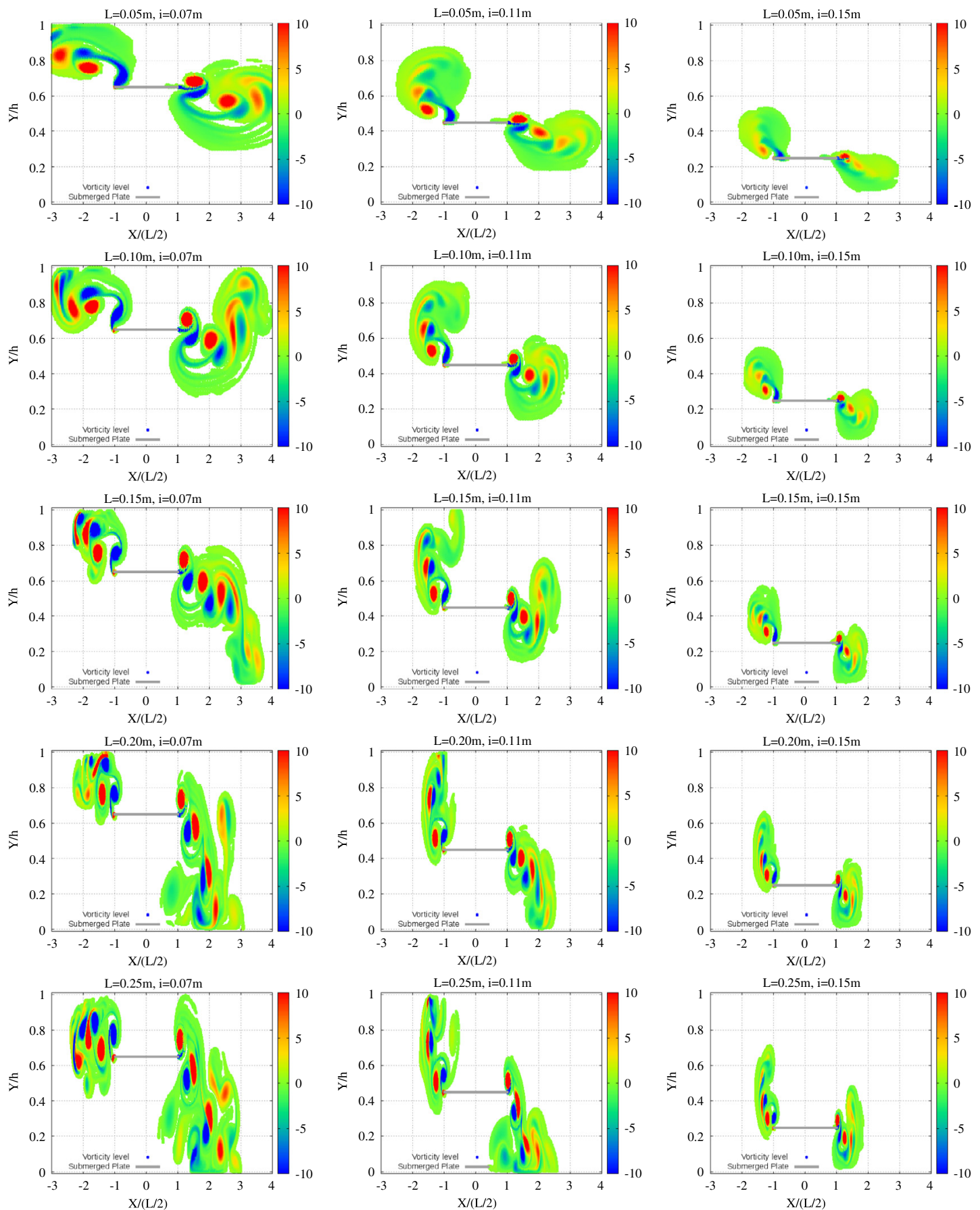

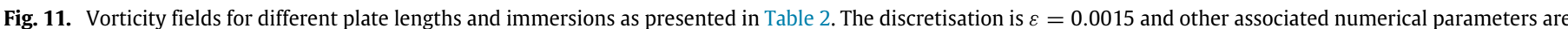
presented in Table 1. All vorticity fields are presented for $t / T=0$, seven wave periods after the initialisation of the computation.

Table 2

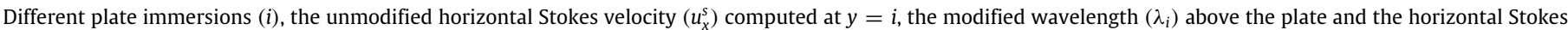
velocity $\left(u_{x}^{s, \lambda_{i}}\right)$ computed at $y=i$ with the modified wavelength $\lambda_{i}$.

\begin{tabular}{|c|c|c|c|c|c|c|c|c|c|c|}
\hline$i(\mathrm{~m})$ & 0.06 & 0.07 & 0.08 & 0.09 & 0.10 & 0.11 & 0.12 & 0.13 & 0.14 & 0.15 \\
\hline$u_{x}^{s}\left(\mathrm{~m} \mathrm{~s}^{-1}\right)$ & 0.071 & 0.069 & 0.067 & 0.065 & 0.064 & 0.062 & 0.061 & 0.060 & 0.059 & 0.058 \\
\hline$\lambda_{i}(\mathrm{~m})$ & 0.736 & 0.790 & 0.833 & 0.883 & 0.924 & 0.962 & 0.997 & 1.030 & 1.061 & 1.090 \\
\hline$u_{x}^{s, \lambda_{i}}\left(\mathrm{~m} \mathrm{~s}^{-1}\right)$ & 0.129 & 0.118 & 0.109 & 0.101 & 0.094 & 0.088 & 0.083 & 0.079 & 0.075 & 0.071 \\
\hline
\end{tabular}

numerical model, based on 2D Lagrangian Vortex method, is developed and applied to this flow configuration, following the preliminary work of Boulier [15]. This method is, by nature, unsteady and well suited for the computation of highly vortical flow in an open domain. The present numerical implementation proved to be accurate in representing the flow characteristics for the two plate lengths ( $L=0.25 \mathrm{~m}$ and $L=0.05 \mathrm{~m}$ ), moderate plate immersions and the given wave characteristics. The numerical velocity and vorticity fields were compared to their experimental counterparts with a good agreement. 


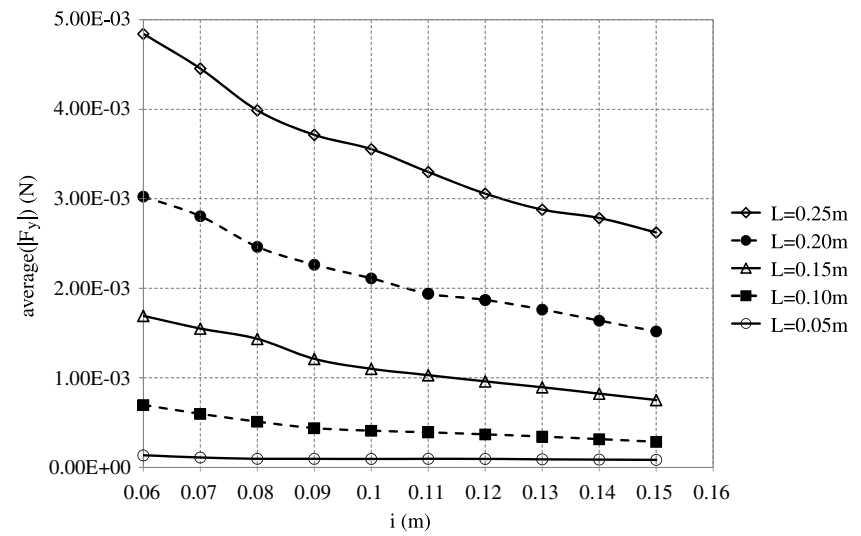

Fig. 12. $\mid \overline{F_{y} \mid}$, the force computed on the plate as a function of the immersion $i$. The different immersions are presented in Table 2; the geometrical and numerical parameters are described in Table 1 . All the computations were run with the finest discretisation of Table 1.

As observed experimentally, the complex flow structure is well reproduced numerically. At each wave period, two pairs of opposite sign vortices are shed one at each edge of the plate. The trajectories of the emitted vortices are also well assessed by the numerical model for the two plate lengths, where comparisons with the experiments can be made. In the smaller plate configuration $(L=0.05 \mathrm{~m})$ the trajectories are slightly better reproduced than for the longer one. In fact, the vortical activity is more intense for the longer plate configuration and more difficult to capture. Additionally, an important recirculation cell is experimentally observed in that configuration at the lower downstream side of the plate. This recirculation cell proved to play an important role on the lee-side vortices trajectories. In fact, the recirculation cell is generated by the impact of the lee-side vortices impacting the seabed. The vortices impact creates a shear flow on the seabed, generating vorticity and eventually feeding the cell. This recirculation cell being created will modify the lee-side vortices trajectories and enhance the impact phenomenon.

As mentioned, the lee-side vortices create a quasi steady jetlike flow which is clearly observable on the averaged flow. This jet-like flow may be a cause of important scour at the jet impact location. This phenomenon is well pronounced for the longer plate configuration ( $L=0.25 \mathrm{~m}$ ), whereas the lee-side vortices hardly impact the seabed for the smaller configuration owing to the lower vortical activity. Numerically speaking, the impact location can be accurately reproduced only with the account of the recirculation cell. Unfortunately, the ad hoc implementation of this cell in the numerical model cannot be applied for all plate lengths and wave characteristics, as experimental information is required. A possible numerical implementation can be addressed in the future in order to cope with this weakness of the present numerical model.

On the upstream side of the plate, the vortices emission is well operated and the vortical patterns are very similar with the experimental ones. In the mean flow, the presence of another large recirculation cell at the lower upstream side is accurately reproduced without any modification of the code. In addition, a smaller cell on top of the previous one and located right above the plate is also observed numerically as well as experimentally. This complex mean flow configuration is well reproduced numerically. The present method, enabling the decomposition of each velocity component, showed that this mean flow is a complex combination of the wave field, the vortical field and the potential velocity owing to the presence of the plate.

Finally, the last section of this paper depicts the force assessment onto the plate. The numerical force evaluation is validated by means of comparisons with simple academic configurations (see Appendix C). Then, the case of the horizontal plate in a wave field

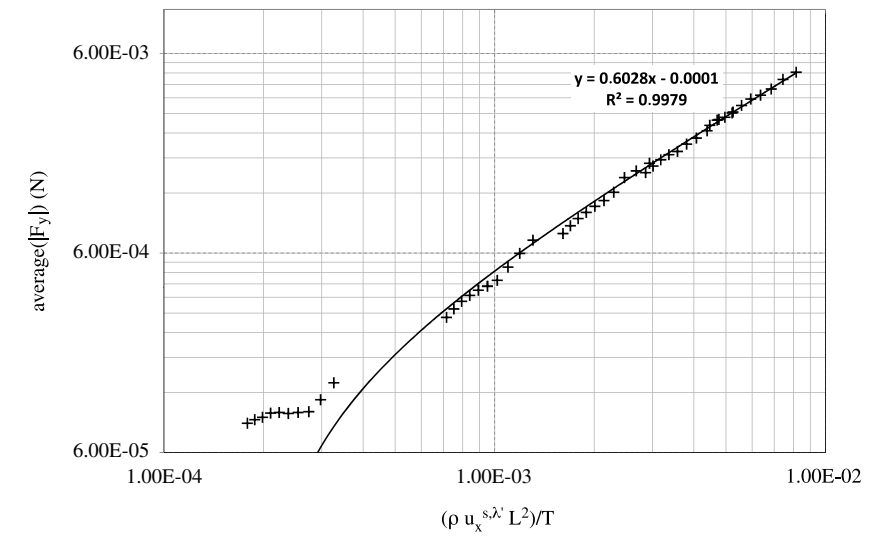

Fig. 13. Forces $\overline{\left|F_{y}\right|}$ acting on the plate as a function of $\rho u_{x}^{s, \lambda_{i}} L^{2} / T$.

is addressed. Regarding these aspects, two main conclusions can be drawn. Firstly, the force recorded on the plate is by far much more important than the Stokes wave induced pressure (and hence force). Secondly, the instantaneous pressure distributions on the plate could be directly correlated to the shed vortices close to the plate.

A parametric study is conducted to analyse the evolution of the forces with respect to the controlling parameters of the flow. Thus the relative immersion $i / h$, with $h$ the total water depth, is varied between 0.3 and 0.75 . Five plate lengths are computed varying from $0.05 \mathrm{~m}$ to $0.25 \mathrm{~m}$ leading to a plate length $\mathrm{L}$ to incident wavelength ratio $L / \lambda$ varying between 0.04 and 0.2 . Owing to the presence of the immersed structure, the wave characteristics are modified above the plate. Thus, a modified wavelength $\lambda_{i}$ and characteristic velocity $u_{x}^{s, \lambda_{i}}$ are determined by considering the first order Stokes model for a water depth equal to the immersion depth. A new scaling is found for lifting forces acting on the plate. Indeed, except for the smallest plate $(L=0.05 \mathrm{~m})$, the forces acting on the plate actually scale with $\rho u_{x}^{s, \lambda_{i}} L^{2} / T$ with a very good accuracy, whatever the immersion is. For a small ratio $L / \lambda$ $(L / \lambda=0.04$ for $L=0.05 \mathrm{~m})$, this scaling does not fit since the wave characteristics are almost not modified by the presence of the plate. But for larger values of $L / \lambda$, the characteristic velocity $u_{x}^{s, \lambda_{i}}$ is the relevant parameter for dimensioning the forces on the plate.

As a perspective, a non linear free surface model similar to the one of Fontaine et al. [39] or more recently Liu et al. [13] is planned in a near future. This will enable a better representation of the flow on the upper part of the plate, between the horizontal plate and the free surface. Additionally, such a model will enable the computation of a wider range of plate length $L$ to wavelength $\lambda$ ratio. Such a non-linear free surface model would enable to increase the value of $L / \lambda$ still in the limit of non-breaking wave configurations. However, a special attention to vortices interactions with the free surface would have to be paid.

Regarding the seabed boundary condition, a no-slip boundary condition can be considered. Possible numerical implementations are those of Ploumhans et al. [38] or Beaudoin et al. [40], keeping in mind that the considered Reynolds number may be difficult to reach. However, the implementation of a no-slip boundary condition is essential in order to capture all the flow structures. The quasi steady recirculation cell situated at the lower downstream side of the plate, and experimentally observed in Poupardin et al. [11], is concerned here.

Finally, these two numerical improvements being achieved, the implementation of a structural model to the plate is possible in order to model flexible membranes immersed in the sea. This type of flexible membrane is currently under development in order to harvest energy from tidal currents, for instance. Some 


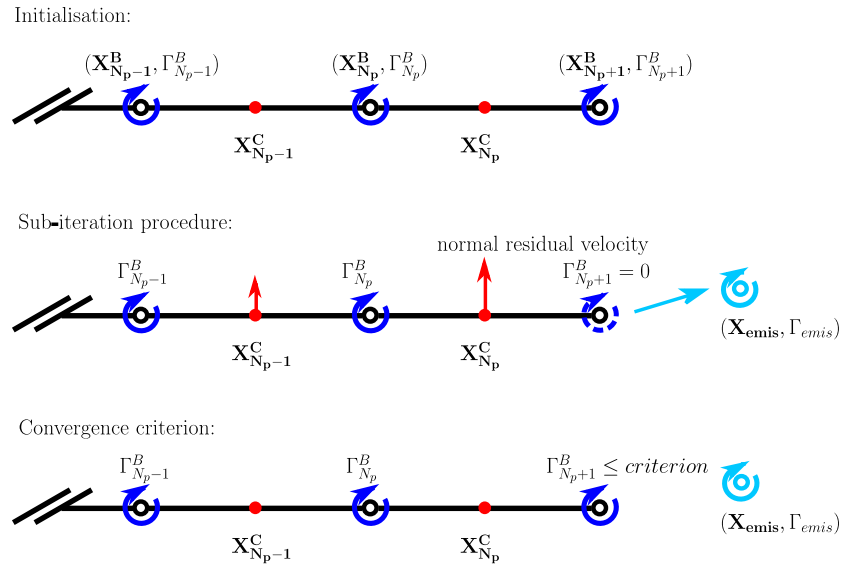

Fig. A.14. Schematic representation of the particles emission process.

physical basis on the technology can be found in the work of Allen et al. [41] and a recent experimental and numerical tests were performed by Deporte et al. [42]. An improvement of the previous numerical implementation is scheduled in order to apply the developed numerical model for current (and wave) interaction with an undulating pre-stressed membrane for energy application. This gives to the software an interesting opportunity for energy applications.

\section{Acknowledgements}

The authors would like to thank the Regional Council of Normandie for their financial support and especially the GRR Energie program named NEPTUNE. The authors also would like to thank the CRIANN (Centre Régional Informatique et d'Applications Numériques de Normandie) for the CPU time provided and their technical support. Finally, the authors also would like to thank the French national program EMACOP (Energie MArine CÔtière et Portuaire) for their financial support for this work.

\section{Appendix A. Particles emission}

To model the vortex particle's emission, an approximation of the Kutta condition is used as presented in [29]. Some theoretical considerations can be found in the textbooks of Lewis [29] and in Katz and Plotkin [30]. Practical details can be found in Nitsche et al. [24,25], Le Maître et al. [27] or Luersen et al. [43]. In the present configuration, the plate is considered here as infinitely thin. In the present approach, the main consideration is to transfer vorticity attached to the flat plate (or bound vortices) $\left(\Gamma_{i}^{B}\right)_{i=1, N_{p}+1}$ into free particles $\left(\Gamma_{j}\right)_{j=1, N_{\omega}}$. his transfer is performed by applying the Kutta condition. In order to detail the emission process, let us start with the scheme described on Fig. 3 of Section 2.4. At each edge of the plate, a bound vortex exists, whose circulation is either $\Gamma_{1}^{B}$ or $\Gamma_{N_{p}+1}^{B}$. This bound vortex is very similar to a free particle defined in Section 2.3, and basically needs to be transferred from the plate to the flow. Whatever the considered edge, this transfer or emission process (detailed in Fig. A.14 for the lee-side edge) can be explained as the following:

1. Initialisation: At the beginning of the emission process, a non null bound vortex $\Gamma_{N_{p}+1}^{B}$ exists at the edge of the plate. Using the tangential component of $\mathbf{U}\left(\mathbf{X}_{N_{p}+1}^{C}\right)$, the normal component is now null owing to the resolution of Eq. (15) of Section 2.5, a new free vortex particle is emitted at a position

$\mathbf{X}_{\text {emis }}=\mathbf{X}_{N_{p}+1}^{B}+0.5 * d t * \mathbf{U}\left(\mathbf{X}_{N_{p}}^{C}\right)$.
$\mathbf{U}\left(\mathbf{X}_{N_{p}}^{C}\right)$ is evaluated with Eq. (18). This new particle $\left(\mathbf{X}_{e m i s}, \Gamma_{e m i s}\right)$ carries a circulation $\Gamma_{\text {emis }}$ equal to the former value of $\Gamma_{N_{p}+1}^{B}$ and $\Gamma_{N_{p}+1}^{B}$ is now set to zero. A new particle is now emitted and the value of $N_{\omega}$ is modified accordingly.

2. Sub-iteration procedure: The free-slip condition (Eq. (13)) is however no more valid, especially at $\mathbf{X}_{N_{p}}^{C}$. In order to enforce this free-slip condition, a new sub-iteration process is engaged, where the matrix system (Eq. (15)) is solved again and where the Kelvin's condition (Eq. (14)) is updated accordingly to the new emitted particle. Eq. (15) being solved, a new non-null value of $\Gamma_{N_{p}+1}^{B}$ is now obtained together with the existence of the emitted particle $\left(\mathbf{X}_{\text {emis }}, \Gamma_{\text {emis }}\right)$. The emitted particle is now updated as the following: its position is re-calculated by means of Eq. (A.1) and its circulation is updated by adding to $\Gamma_{\text {emis }}$ the residual value of $\Gamma_{N_{p}+1}^{B}$. This finalises the sub-iteration procedure.

3. Convergence criterion: This sub-iteration procedure (step 2) is performed again until the residual value of $\Gamma_{N_{p}+1}^{B}$ computed by Eq. (15) is inferior to a user defined criterion. The criterion was set to $10^{-7}$ in all the presented computations, which leads to approximately ten to maximum twenty sub-iteration procedures.

The computation starts without any particle and, at each time step, new particles are created. In the validation case of an infinitely thin air-foil (see Appendix C), only one particle is emitted at the trailing edge of the air-foil at each time step. In the case of the flat plate in a wave field, particles are emitted at both plate edges leading to the creation of two new particles at each emission procedure. However, owing to the regular global remeshing of the flow, many particles are created during this remeshing procedure making the number of particles increase faster than one or two times the number of time steps. To avoid such an increase in the number of particles after each global remeshing, a vorticity threshold must be defined. This threshold was set to $\omega_{\min }=0.1 \mathrm{rad} \cdot \mathrm{s}^{-1}$, which corresponds to less than $1 / 1000$ of the maximum vorticity values recorded in the experiments described in Ref. [11]. This $\omega_{\min }$ was chosen because it allows a complete conservation of the initial circulation $\Gamma_{0}$ of a Lamb-Oseen vortex freely advected over 5 periods of time and initialised with the poorest discretisation used for the computations (see Table 1 of Section 3.1). This Lamb-Oseen vortex is similar to those observed in the lee-side of the flat plate.

\section{Appendix B. Treatment of diffusion and dissipation}

The account of diffusion in Vortex Method can be treated in several manners. Apart from the pioneering method of random walk proposed by Chorin [44], mainly two methods revealed to be sufficiently accurate and efficient, namely the Diffusion Velocity Method (DVM) [45-48] and the Particle Strength Exchange (PSE) method [16-18]. The latter is currently the most commonly used. In the present numerical implementation, the $2 \mathrm{D}$ version of the Particle Strength Exchange proposed by [17] is chosen. This PSE method computes an accurate diffusion of vorticity together with very conservative properties.

However, in order to restore the dissipation process, an artificial dissipation was implemented. Let $t_{0}=0$ be the origin of time. At each time step, the particle's circulation is multiplied by a dissipation coefficient $\beta^{d t}$. At the end of the first time step the circulation is modified:

$\Gamma_{j}\left(t_{0}+d t\right)=\beta^{d t} \Gamma_{j}\left(t_{0}\right)$.

After $n$ time steps, the previous relation becomes:

$\Gamma_{j}\left(t_{0}+n d t\right)=\beta^{n d t} \Gamma_{j}\left(t_{0}\right)$, 

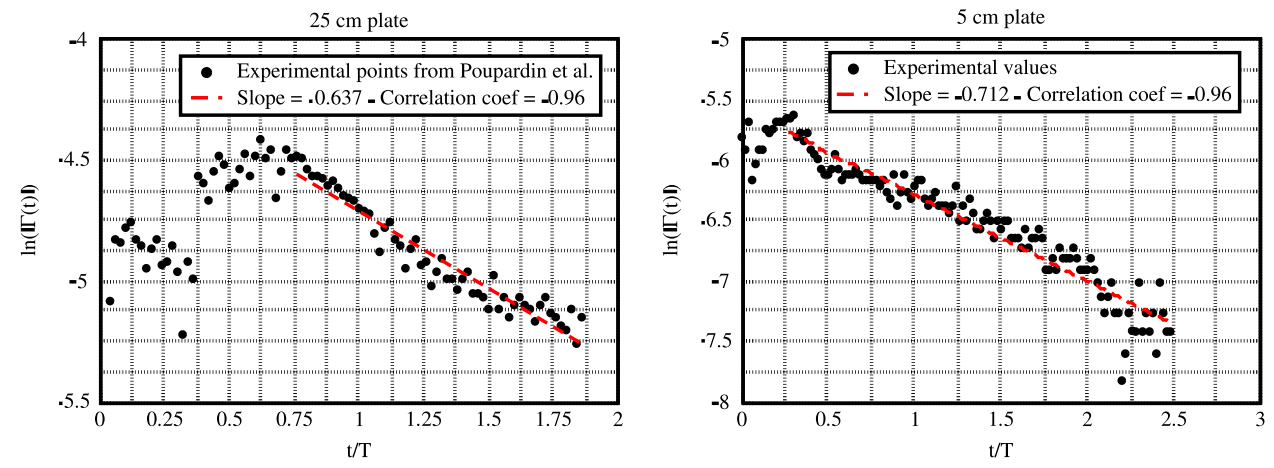

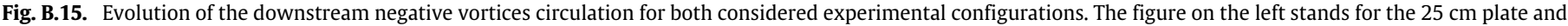
the one on the right stands for the $5 \mathrm{~cm}$ plate. Experimental values were reproduced from Fig. 9 of Poupardin et al. [11] and from Poupardin [33] for the smaller plate.

finally leading to the following relation at any time $t=t_{0}+n d t$ :

$\ln \left(\left|\Gamma_{j}(t)\right|\right)=\ln (\beta) *\left(t-t_{0}\right)+\ln \left(\left|\Gamma_{j}\left(t_{0}\right)\right|\right)$.

With this definition of $\beta$, this parameter physically represents a desired circulation dissipation per second. In order to assess $\beta$, the experimental values of the vortices circulation were reproduced from Fig. 9 of Poupardin et al. [11]. Fig. B.15 depicts the evolution of negative downstream vortices circulation for the two plate lengths ( $L=0.25 \mathrm{~m}$ and $L=0.05 \mathrm{~m}$ ). In both cases, a linear fit of the vortices circulation with natural logarithm $\ln (|\Gamma(t)|)$ is obtained with a correlation coefficient of $\approx 0.96$; a correlation coefficient of 1.0 being an exact linear fit.

From the obtained fitted slopes of Fig. B.15, the value of $\beta$ should approximately be 0.53 for the larger plate $(L=25 \mathrm{~cm})$ and $\beta \approx$ 0.49 for the smaller one $(L=5 \mathrm{~cm})$. This physically means that the vortices circulation is dissipated by $\approx 50 \%$ every second, equivalent to a wave period $T=1 \mathrm{~s}$.

In order to assess the best value for $\beta$, a L2-error based on the difference of location between the experimental and numerical vortices centre was post-treated. Following the experimental approach, a method based on $\lambda_{2}$-criterion (see for instance Jeong et al. [49]) was used to determine the vortices centres together with a method based on the local maximum of vorticity $\omega_{\max }$. The two graphs of Fig. B.16 depict the L2-error evolution for $\beta$-values between 0.50 to 0.825 and for both plates length. As a matter of readability, only the two finer discretisations presented in Table 1 are depicted. $\beta=0.50$ corresponds to $50 \%$ circulation conservation per second and $\beta=0.80$ corresponds to a more conservative situation with $80 \%$ circulation conservation per second.

Whatever the $\beta$-value in the given range, the absolute L2-error values are not excessive, indicating that the downstream vortices centre trajectories are generally correctly reproduced. For the lower values of $\beta$, the vortices dissipation is too important. The downstream vortices being too weak, they are basically advected by the wave velocity component of the flow (see Eq. (6) of Section 2.2). This is probably what happened to the $\varepsilon=0.0030$ trajectory depicted on Fig. 5 (a). For the 0.05 m-plate, the experiments showed that the vortical activity is weaker than for the longer plate. And the vortices have a tendency to follow the wave field. This may explain why the computations generally better reproduce vortex dynamics for this smaller plate (see Fig. B.16(b)).

For the larger plate, the situation is somehow different. Low values of $\beta$ induce a too important dissipation as already mentioned. For higher values, e.g. $0.70 \lesssim \beta \lesssim 0.75$, the vortices activity is enhanced and mutual induction lead to the downward trajectory, as experimentally observed and analysed by Poupardin et al. [11]. For these values, the lee-side vortex pairs are advected downward (see the vorticity field depicted on Fig. 6) and are destroyed after two to three wave periods. On the contrary, from $\beta \gtrsim 0.75$, an increase in L2-error can be observed. In fact, too high values of $\beta$ lead to a more important vortices activity than in reality. The computations are too conservative and, after the vortices have bounced on the wave flume bottom, they spuriously interact with those of previous wave periods. A satisfactory representation of the vortices trajectories was finally favoured. Therefore, a value of $\beta=0.75$ is chosen for all the following computations of the paper as it represents the best compromise between a too dissipative and too conservative situation.

\section{Appendix C. Validation of the force evaluation}

Following the work of Le Maître et al. [27] dealing with a numerical study of two-dimensional sail in unsteady flow, their expression for the determination of the force acting on the plate will be considered. In their study, the fluid is considered inviscid, incompressible and the sail is supposed to have a very small thickness. Their configuration is very similar to the present one, therefore their study and results will be taken as a reference. According to equation (11) of the above-cited paper [27], the pressure jump $\Delta P$ acting on the thin structure (either a sail or a plate) can be calculated thanks to the following equation:

$\Delta P=\rho\left[\gamma^{B} \mathbf{e}_{z} \wedge \mathbf{U}(\mathbf{x})\right] \cdot \mathbf{n}+\rho \int_{0}^{l} \frac{d \gamma^{B}\left(l^{\prime}\right)}{d t} d l$

where $\gamma^{B}$ represents the vortex sheet strength distribution along the plate. It physically represents the tangential velocity jump across the plate and is therefore homogeneous to $\left[\mathrm{m} \cdot \mathrm{s}^{-1}\right]$. The density $\rho$ is going to be set to unity without any loss of generality and n stands for the plate normal vector.

In this Eq. (C.5), the first term stands for the steady component and the second for the unsteady one. For the ith element of the discretised plate, assuming that the line elements are constant and equal to $d \ell_{p}$, and that the $\gamma^{B}$ distribution is piecewise constant along a plate element $d \ell_{p}$; the bound circulation stands for $\Gamma_{i}^{B} \approx$ $\gamma_{i}^{B} d \ell_{p}$. Therefore, the $i$ th component of the force acting on the flat plate $\mathbf{F}_{i}$ can be expressed by multiplying the pressure jump $\Delta P$ at a $\mathbf{X}_{i}^{B}$ location with the line element length $d \ell_{p}$. And finally, the total force $\mathbf{F}$ acting on the plate is:

$\mathbf{F}=\sum_{i=1, N_{p}} \Gamma_{i}^{B} \mathbf{e}_{z} \wedge \mathbf{U}\left(\mathbf{X}_{i}^{B}\right)+\sum_{i=1, N_{p}} \sum_{j=1, i} \frac{d \Gamma_{j}^{B}}{d t} d \ell_{p}$,

where the $\Gamma_{i}^{B}$ and $\frac{d \Gamma_{j}^{B}}{d t}$ values are evaluated on $\mathbf{X}_{i}^{C}$.

Prior to running computations on the desired configuration described on Fig. 2, several cases were tested in order to assess the accuracy of the developed software. The first test case is a flat plate immersed with an incident angle in a flow of constant velocity. This test case is very simple and the computations are compared 


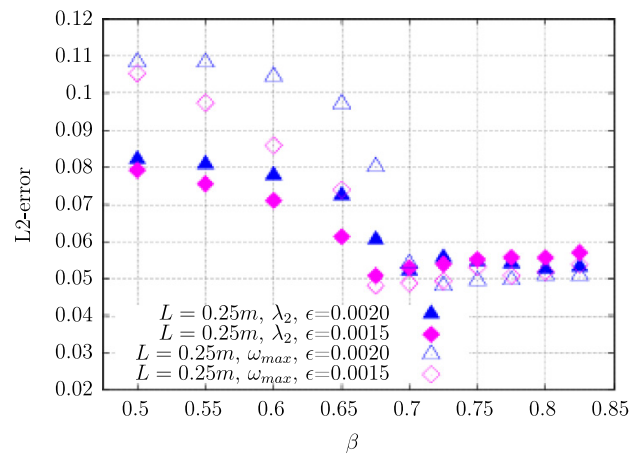

(a) $L=0.25 \mathrm{~m}$.

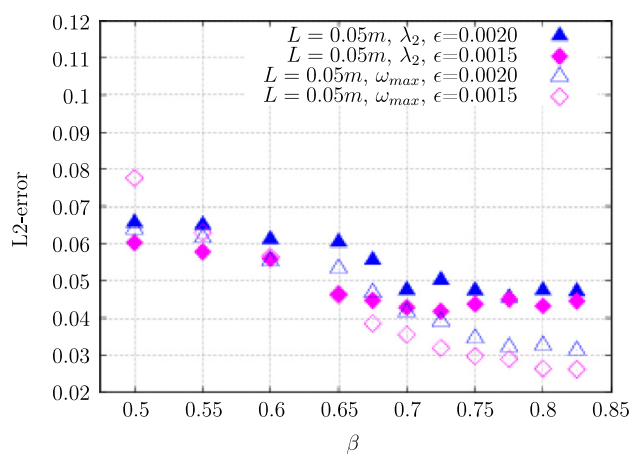

(b) $L=0.05 \mathrm{~m}$.

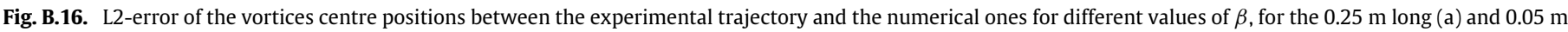
long (b) plates. The $\lambda_{2}$ and $\omega_{\max }$ detection techniques are presented with the two finer discretisations presented in Table 1 of Section 3.1.

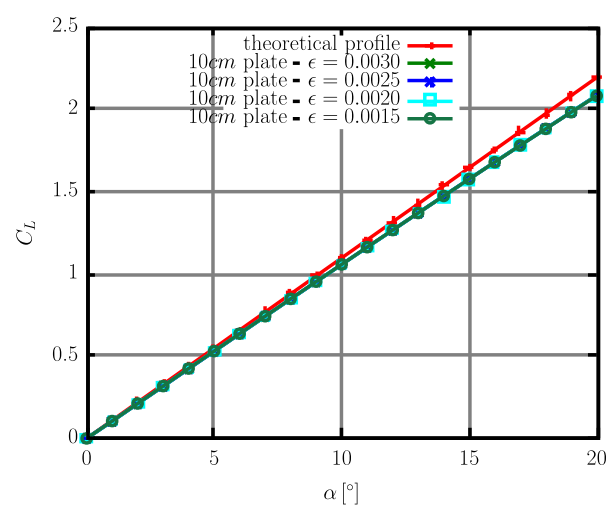

(a) Static case for validation of the steady component (Eq. (C.6)) of the force.

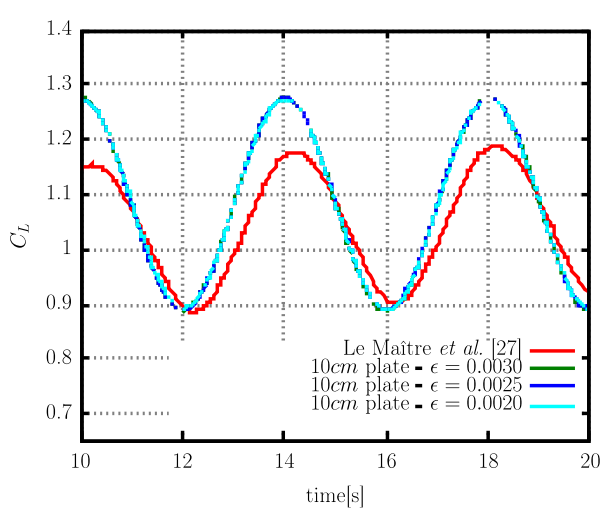

(b) Oscillating case for validation of the unsteady component (Eq. (C.6)) of the force.

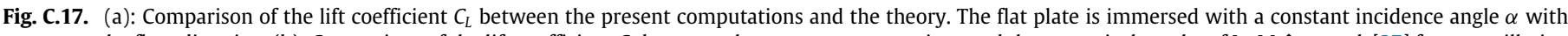

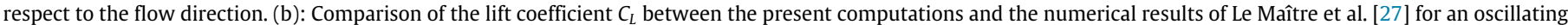

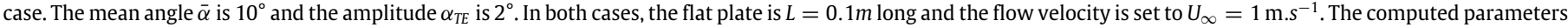
are described in Table 1 for both cases.

with analytical results. Secondly, in order to completely assess the force evaluation, and especially its unsteady component, a second test case based on the numerical study of Le Maître et al. [27] is reproduced.

First, in order to validate the steady component (Eq. (C.6)) of the force, a $10 \mathrm{~cm}$ flat plate immersed with a constant incident angle $\alpha$ with respect to the flow direction is considered. The flow velocity is set to $U_{\infty}=1 \mathrm{~m} \cdot \mathrm{s}^{-1}$. The lift coefficient $C_{L}$ is defined as follow [29]:

$C_{L}=\frac{\mathbf{F} \cdot \mathbf{n}}{0.5 \rho U_{\infty}^{2} L}$.

For such a configuration and in the infinitely thin air-foil theory $[29,30]$, the theoretical lift coefficient $C_{L}$ is supposed to be equal to $2 \pi \frac{\alpha}{180}$ whatever the incident angle is, provided that the incident angle remains small. In fact, the incident angle is supposed to be small enough to avoid stall, which is generally the case for a thin air-foil with low incident angle. Fig. C.17(a) presents the numerical results for the 4 discretisations described in Table 1 and the theoretical $C_{L}$ values. All the computations were run inviscid on a sufficiently long duration in order to reach a steady state. From Fig. C.17(a), one can observe that our results are in good agreement with the theory. For the lower angle of attack $\left(\alpha \lesssim 7^{\circ}\right)$, the numerical results quasi superimpose with the theory. And the more $\alpha$ increases, the more the numerical results deviate from the theoretical values, which was expected as the thin air-foil theory hypothesises low angles of attack. However, the four presented discretisations exactly superimpose and the results are converged. This validates the numerical implementation of the steady component.
The final aim is to evaluate the force on a plate in a unsteady wave field, the unsteady component of Eq. (22) also needs to be assessed. In that purpose, the same configuration as the one proposed by Le Maître et al. [27] is used. It is basically a flat plate of length $L=10 \mathrm{~cm}$, which is fixed at its upstream edge and waved around a mean incident angle $\bar{\alpha}=10^{\circ}$ with an amplitude of $2^{\circ}$. Fig. C.17(b) depicts the lift coefficient $C_{L}$ with respect to time for the numerical results reproduced from Figure 3 of Le Maître et al. [27] and three out of the four presented discretisation. The finer discretisation $\varepsilon=0.0015$ was not computed here as the three roughest ones were already completely converged and superimposed. It is worth indicating that there actually is a typo in Ref. [27] on the caption of Fig. 3, where a mean angle $\bar{\alpha}=10^{\circ}$ should be considered. The presented numerical results are similar to those of Ref. [27] although some discrepancies can be identified. Several explanations can be argued in order to justify these differences. The first one is that the structure is not fully rigid, it is in fact deformable, in Le Maître et al. study, as it is supposed to reproduce a sail. This may partially explain the higher amplitude found in the present results and the short phase shift on the extrema. Additionally, in the present numerical implementation, a global remeshing of the flow is considered which enhances the quality of the results and the discretisation is much finer. Finally, it is clearly indicated in their paper that the $C_{L}$-value is slightly increasing with time and that a mean steady value of the lift coefficient $C_{L}$ was not reached. In the presented results of Fig. C.17(b), a steady mean value of $C_{L}=1.05$ is reached for the presented periods of time. This mean value actually corresponds to the $C_{L}$-value with $\bar{\alpha}=10^{\circ}$ of the steady configuration (cf. Fig. C.17(a)). As a conclusion, the present numerical implementation of Eq. (22) is validated with these two test cases. 


\section{References}

[1] P.F. Siew, D.G. Hurley, Long surface waves incident on a submerged horizontal plate, J. Fluid Mech. 83 (1977) 141-151.

[2] M. Patarapanich, Maximum and zero reflection from submerged plate, J. Waterway Port Coastal Ocean Eng. 110 (2) (1984) 171-181.

[3] R. Usha, T. Gayathri, Wave motion over a twin-plate breakwater, Ocean Eng. 32 (2005) 1054-1072.

[4] M. Parapatanich, H.F. Cheong, Reflexion and transmission characteristics of a regular and random wave from a submerged horizontal plate, Coastal Eng. 13 (1989) 161-182.

[5] J. Brossard, G. Perret, L. Blonce, A. Diedhiou, Higher harmonics induced by a submerged horizontal plate and submerged rectangular step in a wave flume Coastal Eng. 56 (2009) 11-22.

[6] F.C.K. Ting, Y.K. Kim, Vortex generation in water waves propagating over a submerged obstacle, Coastal Eng. 24 (1994) 23-49.

[7] K.A. Chang, T.J. Hsu, P.L.F. Liu, Vortex generation and evolution in water waves propagating over a submerged rectangular obstacle. part i : Solitary waves, Coastal Eng. 44 (2001) 13-36.

[8] K.A. Chang, T.J. Hsu, P.L.F. Liu, Vortex generation and evolution in water waves propagating over a submerged rectangular obstacle. part ii : Cnoidal waves, Coastal Eng. 52 (2005) 257-283.

[9] C. Lin, T.C. Ho, S.C. Chang, S. C, K.A.Chang Hsieh, Vortex shedding induced by a solitary wave propagating over a submerged vertical plate, Int. J. Heat Fluid Flow 26 (2005) 894-904.

[10] J.-M. Cherfils, L. Blonce, G. Pinon, E. Rivoalen, Simulation of water wave coastal structure interaction by smoothed particle hydrodynamics, in: 8th International Conference on Hydrodynamics (ICHD), 2008, Nantes, France.

[11] A. Poupardin, G. Perret, G. Pinon, N. Bourneton, E. Rivoalen, J. Brossard, Vortex kinematic around a submerged plate under water waves. Part I: Experimental analysis, Eur. J. Mech. B Fluids 34 (0)(2012) 47-55. http://dx.doi. org/10.1016/j.euromechflu.2012.02.003, URL http://www.sciencedirect.com/ science/article/pii/S0997754612000349.

[12] V. Rey, J. Touboul, Forces and moment on a horizontal plate due to regular and irregular waves in the presence of current, Appl. Ocean Res. 33 (2011) 88-99.

[13] C. Liu, Z. Huang, S.K. Tan, Nonlinear scattering of non-breaking waves by submerged horizontal plate : Experiments and simulations, Ocean Eng. 36 (2009) 1332-1345.

[14] J.-M. Cherfils, Développements et applications de la méthode sph aux écoulements visqueux à surface libre (Ph.D. thesis), Université du Havre, 2011.

[15] B. Boulier, Evolution spatio-temporelle de structures tourbillonnaires dans la houle (Ph.D. thesis), Université du Havre, 1996.

[16] P. Degond, S. Mas-Gallic, The weighted particle method for convection-diffusion equations. Part I: The case of an isotropic viscosity, Math. Comp. 53 (188) (1989) 485-507. http://dx.doi.org/10.2307/2008716.

[17] J. Choquin, S. Huberson, Particles simulation of viscous flow, Comput. Fluids 17 (2) (1989) 397-410. http://dx.doi.org/10.1016/0045-7930(89)90049-2, URL http://www.sciencedirect.com/science/article/pii/0045793089900492.

[18] G. Cottet, P. Koumoutsakos, Vortex Methods: Theory and Practice, Cambridge University Press, 2000.

[19] R.G. Dean, R.A. Dalrymple, Water Wave Mechanics for Engineers and Scientists, World Scientific Publishing Company, 1991.

[20] C. Rehbach, Calcul numérique d'écoulements tridimensionnels instationnaires avec nappes tourbillonaires, Rech. Aérosp. 5 (1977) 289-298.

[21] A. Leonard, Vortex methods for flow simulation, J. Comput. Phys. 37 (3) (1980) 289-335. http://dx.doi.org/10.1016/0021-9991(80)90040-6, URL http://www.sciencedirect.com/science/article/B6WHY-4DD1RFV-1T/2/ 68d5c3a0ce2f03f137353c200b9df3e8.

[22] G.-H. Cottet, P.D. Koumoutsakos, Vortex Method : Theory and Practice Cambridge University Press, UK, 2000

[23] G.S. Winckelmans, A. Leonard, Contributions to vortex particle methods for the computation of three-dimensional incompressible unsteady flows, J. Comput. Phys. 109 (2) (1993) 247-273. http://dx.doi.org/10. 1006/jcph.1993.1216, URL http://www.sciencedirect.com/science/article/ B6WHY-45P11X4-9/2/18db4eee303171d83ce200e0170c4543.

[24] M. Nitsche, Axisymmetric vortex sheet roll-up (Ph.D. thesis), University of Michigan, 1992
[25] M. Nitsche, R. Krasny, A numerical study of vortex ring formation at the edge of a circular tube, J. Fluid Mech. 276 (139-161) (1994).

[26] J. Bousquet, Aérodynamique : Méthode des singularités, Cépaduès Editions, in: Collection La chevêche, 1990, URL http://books.google.fr/books?id= I8X3PAAACAAJ.

[27] O. Le Maître, S. Huberson, E. Souza De Cursi, Unsteady model of sail and flow interaction, J. Fluids Struct. 13 (1999) 37-59.

[28] P.G. Saffman, Vortex Dynamics, Cambrige University Press, 1992.

[29] R. Lewis, Vortex Element Methods for Fluid Dynamic Analysis of Engineering Systems, Cambridge University Press, 1991.

[30] J. Katz, A. Plotkin, Low-Speed Aerodynamics, Cambridge University Press, 2001.

[31] G. Pinon, P. Mycek, G. Germain, E. Rivoalen, Numerical simulation of the wake of marine current turbines with a particle method, Renew. Energy 46 (0) (2012) 111-126. http://dx.doi.org/10.1016/j.renene.2012.03.037, URL http://www.sciencedirect.com/science/article/pii/S0960148112002418.

[32] K. Lindsay, R. Krasny, A particle method and adaptive treecode for vortex sheet motion in three-dimensional flow, J. Comput. Phys. 172 (2001) 879-907.

[33] A. Poupardin, Dynamique tourbillonnaire - application à l'impact environnemental de structures immergées (Ph.D. thesis), Université du Havre, 2012.

[34] K.U. Graw, Shore protection and electricity by submerged plate wave energy converter, in: European Wave Energy Symposium, 1993, pp. 1-6.

[35] K.U. Graw, The submerged plate wave energy converter. a new type of wave energy device, in: ODEC, 1993, pp. 1-4.

[36] R.W. Carter, Wave energy converters and submerged horizontal plate (Ph.D. thesis), University of Hawaii, 2005

[37] G. Orer, A. Ozdamar, An experimental study on the efficiency of the submerged plate wave energy converter, Renew. Energy. 32 (2007) 1317-1327.

[38] P. Ploumhans, G. Winckelmans, Vortex methods for high resolution simulations of viscous flow past bluff-bodies of general geometry, J. Comput. Phys. 165 (2000) 354-406.

[39] E. Fontaine, Simulation de l'écoulement potentiel engendré par un corps élancé perçant la surface libre à fort nombre de froude (Ph.D. thesis), Ecole nationale des ponts et chaussées, 1996.

[40] A. Beaudoin, S. Huberson, E. Rivoalen, From Navier-Stokes to stokes by means of particle methods, J. Comput. Phys. 214 (1) (2006) 264-283. http://dx.doi. org/10.1016/j.jcp.2005.09.014, URL http://www.sciencedirect.com/science/ article/pii/S0021999105004341.

[41] J. Allen, A. Smits, Energy harvesting EEL, J. Fluids Struct. 15 (3-4) (2001) 629-640. http://dx.doi.org/10.1006/jfls.2000.0355, URL http://www. sciencedirect.com/science/article/pii/S0889974600903554.

[42] A. Deporte, M. Trasch, G. Germain, P. Davies, J. Drevet, Study of a flexible membrane current energy converter, in: 10th EWTEC 2013 - European Wave and Tidal Energy Conference Series, 2013, Aalborg, Denmark.

[43] M. Luersen, R. Le Riche, D. Lemosse, O. Le Maître, A computationally efficient approach to swimming monofin optimization, Struct. Multidiscip. Optim. 31 (6) (2006) 488-496. http://dx.doi.org/10.1007/s00158-006-0001-6, 9. URL https://hal.archives-ouvertes.fr/hal-00293057.

[44] A. Chorin, Numerical study of slightly viscous flow, J. Fluid Mech. 57 (1973) $785-796$.

[45] J. Fronteau, P. Combis, A lie admissible method of integration of folklerplank equations with non linear coefficients (exact and numerical solutions), Hadronic J. 7 (1984) 911-930.

[46] Y. Ogami, T. Akamatsu, Viscous flow simulation using the discrete vortex model-the diffusion velocity method, Comput. Fluids 19 (3-4) (1991) 433-441. http://dx.doi.org/10.1016/0045-7930(91)90068-S, URL http:// www.sciencedirect.com/science/article/pii/004579309190068S.

[47] P. Mycek, G. Pinon, G. Germain, Élie Rivoalen, A self-regularising DVM-PSE method for the modelling of diffusion in particle methods, C. R. Méc. 341 (9-10) (2013) 709-714. http://dx.doi.org/10.1016/j.crme.2013.08.002, URL http://www.sciencedirect.com/science/article/pii/S1631072113001137.

[48] P. Mycek, G. Pinon, G. Germain, E. Rivoalen, Formulation and analysis of a diffusion-velocity particle model for transport-dispersion equations, Comput. Appl. Math. (2014) 1-27. http://dx.doi.org/10.1007/s40314-014-0200-5.

[49] J. Jeong, F. Hussain, On the identification of a vortex, J. Fluid Mech. 285 (1995) 69-94. http://dx.doi.org/10.1017/S0022112095000462. 